\title{
6,7-dimethoxy-1,2,3,4-tetrahydro- isoquinoline-3-carboxylic acid attenuates heptatocellular carcinoma in rats with NMR-based metabolic perturbations
}

\begin{abstract}
Aim: 6,7-dimethoxy-1,2,3,4-tetrahydro-isoquinoline-3-carboxylic acid (M1) was synthesized and evaluated for in-vivo antiproliferative action in diethylnitrosamineinduced hepatocarcinogenic rats. Materials \& methods: The antiproliferative effect of M1 was assessed by various biochemical parameters, histopathology of liver and HPLC analysis. Proton nuclear magnetic resonance-based serum metabolic study was implemented on rat sera to explore the effects of $\mathrm{M} 1$ on hepatocellular carcinomainduced metabolic alterations. Results: M1 showed protective action on liver and restored the arrangement of liver tissues in normal proportion. HPLC analysis displayed a good plasma drug concentration after its oral administration. Score plots of partial least squares discriminate analysis models exhibited that M1 therapy ameliorated hepatocellular carcinoma-induced metabolic alterations which signified its antiproliferative potential. Conclusion: M1 manifested notable antiproliferative profile, and warrants further investigation for future anticancer therapy.
\end{abstract}

Lay abstract: Our previous study unveiled that an isoquinoline alkaloid (M1) isolated from Mucuna pruriens seeds had potent antiproliferative action on human hepatoma cells in vitro. In the present investigation we synthesized M1 and studied its in vivo antiproliferative effects in liver carcinogenic rats. To observe the effects of M1, various studies were conducted which include biochemical parameters of plasma and serum, histopathology of liver and HPLC analysis of plasma. In addition, ${ }^{1} \mathrm{H}-\mathrm{NMR}$-based serum metabolic profiling was carried out to identify the effects of M1 on hepatocellular carcinoma-induced metabolic alterations. Our findings showed that compound M1 exemplifies remarkable efficacy against hepatocellular carcinoma.

First draft submitted: 17 January 2017; Accepted for publication: 24 March 2017; Published online: 12 May 2017

Keywords: 6,7-dimethoxy-1,2,3,4-tetrahydro-isoquinoline-3-carboxylic acid

- diethylnitrosamine • hepatocellular carcinoma • NMR-based metabolomics

Hepatocellular carcinoma (HCC) is considered the sixth most common malignancy and third most frequent leading cause of death [1]. It increases risk of death in developing countries, and 7.6 million people died from this cancer according to the WHO report in 2008 [2]. This is a most challenging situation for healthcare institutions as demand is increased for hepatic cancer treatment day to day with consequent economic repercus- sions. Drugs used for cancer chemotherapy are relatively toxic in nature and develop resistance during therapy. The molecular mechanisms of drug resistance may involve a variety of factors such as mutation of target genes and decreased drug concentrations in the cells due to renal toxicity [3-5]. Drugs from a natural origin are very few in number and synthetic drugs are costly and highly toxic to human body. Synthetic chemothera-
Pranesh Kumar ${ }^{\ddagger 1}$, Ashok K Singh ${ }^{\ddagger 1}$, Vinit Raj', Amit Rai', Siddhartha Maity², Atul Rawat ${ }^{3,4}$, Umesh Kumar ${ }^{3}$, Dinesh Kumar ${ }^{3}$, Anand Prakash ${ }^{4}$, Anupam Guleria*,3 \& Sudipta Saha**,1 'Department of Pharmaceutical Sciences, Babasaheb Bhimrao Ambedkar University, Vidya Vihar, Raibareli Road, Lucknow 226025, India

2Department of Pharmaceutical Technology, Jadavpur University, Kolkata 700032

${ }^{3}$ Centre of Biomedical Research, SGPGIMS Campus, Raebareli Road, Lucknow 226014, Uttar Pradesh, India ${ }^{4}$ Department of Biotechnology, Babasaheb Bhimrao Ambedkar University, Vidya Vihar, Raibareli Road, Lucknow 226025, India

*Author for correspondence: anuguleriaphy@gmail.com

**Author for correspondence: sudiptapharm@gmail.com

${ }^{\ddagger}$ Authors contributed equally 
peutic agents have been of proven efficacy for HCC treatment due to their chemoresistance [6]. Therefore, it is necessary to explore some newer anticancer drugs from plant sources to improve cancer prognosis and survival rate.

Natural products have been used for the treatment of hepatic cancer from ancient times but very few compounds isolated till date from natural origin are effective against hepatic cancer [7]. Our previous studies demonstrated that the ethyl acetate and methanol extracts of Mucuna pruriens (MP) seeds have potent antiproliferative action on human hepatoma cell line (Huh-7 cells) [8]. Recent investigation revealed that the seeds of MP are rich with isoquinoline alkaloids [9] and these alkaloids have a dramatic role in anticancer therapy $[10,11]$. Later, our group isolated one isoquinoline alkaloid from MP seeds (namely M1, 6,7-dimethoxy1,2,3,4-tetrahydro-isoquinoline-3-carboxylic acid, Supplementary Figure 1) and demonstrated that M1 had dramatic role in antiproliferation against Huh-7 cells in vitro [12].

However, the in vivo antiproliferative action of M1 has not been reported thus far. Therefore, the present study was undertaken to find out the in vivo antiproliferative effect of M1 in hepatocarcinogenic albino Wistar rats. Various oxidative stress parameters, scanning electron microscopic (SEM) and histopathological studies of the liver tissues were performed to evaluate the protective effect of M1. ${ }^{1} \mathrm{H}$ nuclear magnetic resonance (NMR)-based serum metabolic profiling was also performed to discover the metabolite modulation in HCC rats treated with M1.

\section{Materials \& methods}

Drugs \& reagents

Di-tert-butyl dicarbonate, sodium borohydride, $p$-toluene sulphonic acid and anhydrous methanol were procured from SD Fine Chemicals (Mumbai, India). Polyphosphoric acid and anhydrous methanol were purchased from Loba Chemicals (New Delhi, India). $p$-Iodoaniline, diethylnitrosamine (DEN) and 2,4-dinitrophenylhydrazine were procured from Sigma-Aldrich (Bengaluru, India). ALT and AST kit was acquired from the Transasia Bio-Medicals Pvt Ltd (Baddi, India). All other chemicals were obtained from Himedia (Mumbai, India). All the solvents and chemicals were of analytical grades with $99 \%$ purity and in house distilled water was used throughout the experiment.

\section{Procedure for synthesis of M1}

Synthesis of 2-tert-butoxycarbonylamino-3-(3,4-

dimethoxy-phenyl)-propionic acid (C1)

A solution of di-tert-butyl dicarbonate $(3.4 \mathrm{ml}, 15 \mathrm{mmol})$ in chloroform $(10 \mathrm{ml})$ was added dropwise to a solu- tion of 2-amino-3-(3,4-dimethoxyphenyl)-propionic acid $(3.3 \mathrm{~g}, 15 \mathrm{mmol})$ in chloroform $(10 \mathrm{ml})$ at $0^{\circ} \mathrm{C}$. The mixture was stirred for another $24 \mathrm{~h}$ at room temperature and then it was concentrated to dryness. The residue was a colorless solid (Supplementary Figure 1; C1, 2.8 g, 84.90\% yield).

Synthesis of 2-amino-3-(2-formyl-4,5-dimethoxyphenyl)-propionic acid (C2)

C1 $(2.6 \mathrm{~g}, 8 \mathrm{mmol})$, formic acid $(1.2 \mathrm{ml}, 30 \mathrm{mmol})$ and polyphosphoric acid $(1.03 \mathrm{ml}, 20 \mathrm{mmol})$ were taken in round bottom flask and heated at $60^{\circ} \mathrm{C}$ for $1 \mathrm{~h}$. Later, the reaction mixture was kept at $4^{\circ} \mathrm{C}$ for overnight. A white colored precipitate was obtained (Supplementary Figure 1; C2, $1.67 \mathrm{~g}, 64.23 \%$ yield), filtered and dried.

Synthesis of 2-amino-3-(2-hydroxymethyl-4,5dimethoxy-phenyl)-propionic acid (C3)

A mixture of C2 (1.4 g, 5.53mmol), sodium borohydride $(0.41 \mathrm{~g}, 11.0 \mathrm{mmol})$ and anhydrous methanol $(20 \mathrm{ml})$ was heated under nitrogen atmosphere for $2 \mathrm{~h}$ and then cooled at room temperature. The resulting crystal was filtered, washed with cold methanol $(30 \mathrm{ml})$ and dried under vacuum at $40^{\circ} \mathrm{C}$ to get a white solid (Supplementary Figure 1; C3, $1.2 \mathrm{~g}, 85 \%$ yield).

Synthesis of 6,7-dimethoxy-1,2,3,4-

tetrahydroisoquinoline-3-carboxylic acid (M1)

A mixture of C3 (1.1 g, $4.31 \mathrm{mmol})$ and $p$-toluene sulphonic acid $(0.741 \mathrm{~g}, 4.31 \mathrm{mmol})$ in methanol were taken in $50 \mathrm{ml}$ round bottom flask and stirred for $1 \mathrm{~h}$ at room temperature. Then, the reaction mixture was heated for $5 \mathrm{~h}$ and kept at $4^{\circ} \mathrm{C}$ for overnight. A white color precipitate was obtained which was recrystallized with methanol (Supplementary Figure 1; M1, $0.9 \mathrm{~g}$, $81 \%$ yield).

\section{General experimental procedures for characterization of $\mathrm{M} 1$}

UV spectrum was measured using UV-visible spectrophotometer (Cary-50 Bio, Varian Melbourne, Australia). Infrared spectrum (IR) was recorded in a FT-IR Spectrometer (Nicolet ${ }^{\text {IM }}$ iS ${ }^{\text {TM }} 50$, MA, USA). Spectra from NMR spectroscopy were recorded using $800 \mathrm{MHz}$ NMR spectrometer (Bruker, Rheinstetten, Germany) processed in Topspin-2.1. Direct-infusion mass spectroscopy data were acquired using a hybrid triple quadrupole linear ion trap mass spectroscopy equipped with an electrospray ionization source (2000 QTRAP, Applied Biosystems, CA, USA).

\section{Experimental animals}

Male albino Wistar rats $(80-120 \mathrm{~g})$ were used for this experiment and Institutional Animal Ethical Com- 

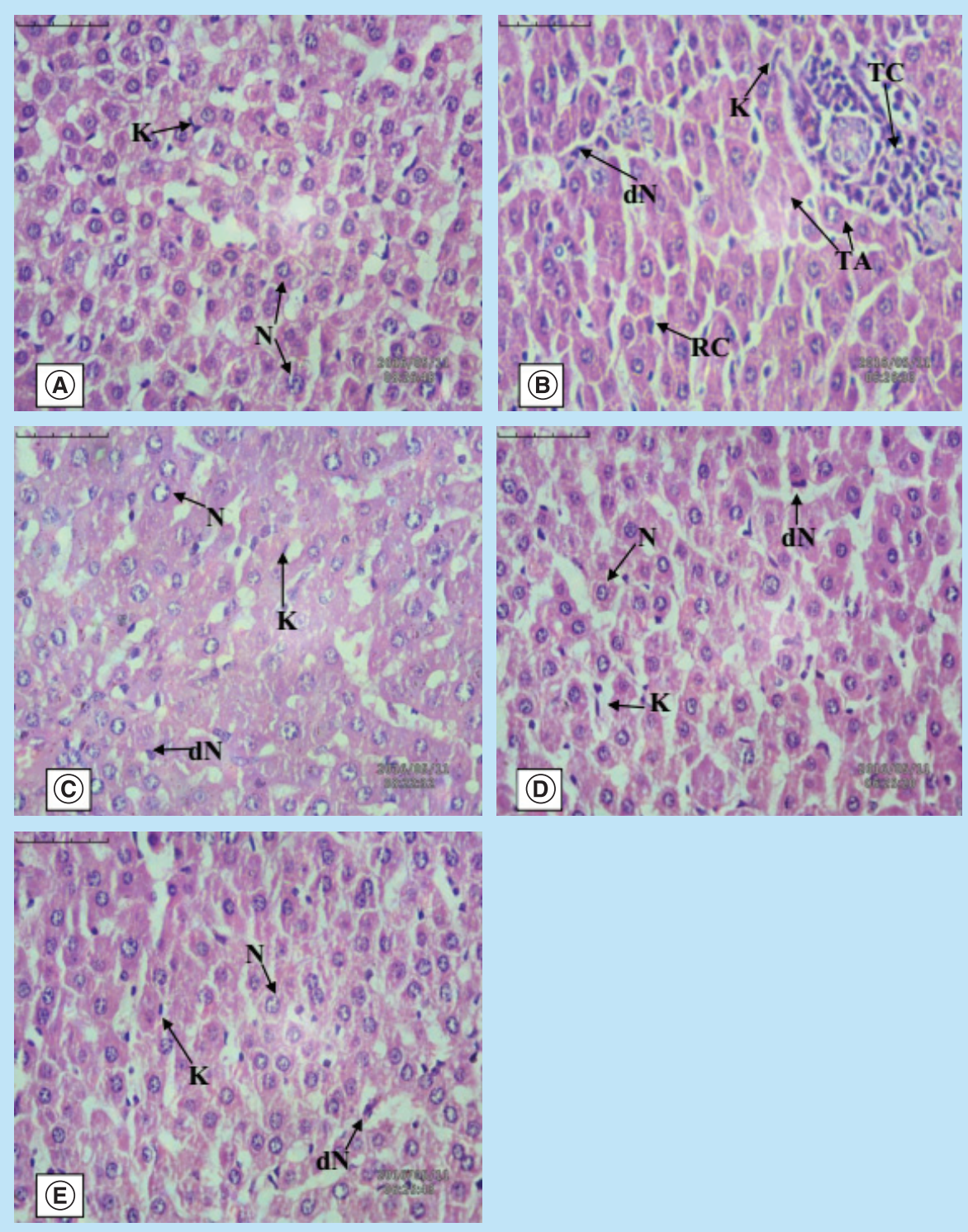

Figure 1. The hepatic pathological changes in diethylnitrosamine-induced hepatocellular carcinoma rats (40X). (A) Normal control, (B) toxic control (DEN), (C) positive control (DEN+5FU), (D) DEN+M1 (50 mg/kg),

(E) DEN+M1 (100 mg/kg). We observed tumor cells, tumor anaplastic cells, dN and ruptured hepatic cell of Kupffer cell for DEN-treated rats. The normal tissue architecture was seen after oral administration of M1.

mittee approved the protocol previously (Approval No. SDCOP\&VS/AH/CPCSE01/017/R2). Standard laboratory conditions (temperature $25 \pm 5^{\circ} \mathrm{C}$ and light/ dark cycle of $12 \mathrm{~h}$ ) were maintained with free access to commercial pellet diet and water ad libitium. Animals were kept for 1 week before experiment.

\section{Acute oral toxicity study}

Acute oral toxicity study of M1 was performed as per revised Organization for Economic Cooperation and Development Guidelines 423. Animal Ethical Committee approved this study (Approval No. SDCOP\&VS/AH/CPCSE01/017/R2). M1 was dis- solved in $0.25 \%$ carboxymethyl cellulose (CMC) and administered orally at the dose of 25, 50, 100, $250 \mathrm{mg} / \mathrm{kg}$ body weight to albino Wistar rats for 4 weeks $(n=6)$ and the animals were observed every day for any toxic manifestations.

\section{Experimental design}

All animals were randomly divided into five groups of six animals each. Drugs were suspended in $0.25 \%$ CMC, subjected to 28 days' treatment orally and divided as follows: Group I: $0.25 \%$ CMC $(2 \mathrm{ml} /$ $\mathrm{kg}$ ), Group II: DEN (8 mg/kg, intraperitoneally) [13], Group III: DEN+5-fluorouracil (5-FU, $10 \mathrm{mg} / \mathrm{kg}$, 
intraperitoneally), Group IV: DEN+M1 $(50 \mathrm{mg} / \mathrm{kg}$, orally) and Group V: DEN+M1 (100 mg/kg, orally). The procedure to induce HCC was adopted from a paper by Song et al. [13] where it is shown that DEN produces $\mathrm{HCC}$ at $8 \mathrm{mg} / \mathrm{kg}$ intraperitoneal injection for 28 days to albino Wistar rats. After initial 1 week of adaptive inhabitation, all rats of group II-V were administered with DEN ( $8 \mathrm{mg} / \mathrm{kg}$, intraperitoneally) for 28 days. After 28 days, 5-FU and M1 were given for 15 days as mentioned above for group III-V. At the end of the experimental period, animals were sacrificed by cervical decapitation and livers were dissected out immediately, rinsed in ice cold saline and stored at $-20^{\circ} \mathrm{C}$ for further studies. The serum was collected, processed and stored for further analysis.

It should be noted that we have performed the in vivo experiments at 50 and $100 \mathrm{mg} / \mathrm{kg}$ doses as M1 is naturally isolated compound and usually $1 / 5$ th, and 1/10th of the safe dose is used for the in vivo studies (determined from acute toxicity studies using Organization for Economic Cooperation and Development Guidelines). Further, in most of the published papers on the naturally occurring compounds, the in vivo studies on albino Wistar rats were carried out at 50 and $100 \mathrm{mg} / \mathrm{kg}$ bodyweight doses [14,15].

\section{Estimation of plasma aspartate \\ aminotransferase, alanine aminotransferase, biochemical estimations \& bilirubin, biliverdin in liver \\ Liver function biomarkers like aspartate aminotrans- ferase (AST), alanine aminotransferase (ALT) were also measured in serum using commercially available kit from Ebra Diagonesis Pvt Ltd, Germany [14]. The oxidative parameters like superoxide dismutase (SOD), thiobarbituric acid reactive substances (TBARS) [16], protein carbonyl (PC) [17], SOD [14], tissue catalase (CAT) [16] and glutathione (GSH) [18] levels were esti- mated in liver tissue in the similar experiment. The total protein content of each sample was measured using the Bradford reagent and bovine serum albu- min was used as a standard. Conjugated bilirubin and biliverdin in liver were measured as per the following procedure stated earlier in the literature with slight modifications [19].}

\section{Histopathological studies}

Histopathological studies were also performed to find out the morphological changes of liver cells after M1 administration [20]. Liver tissues from each group were assessed for their morphological changes using hematoxylin and eosin staining. The tissues were preserved in $10 \%$ formalin overnight. Next day, the tissues again were superseded by $70 \%$ isopropanol overnight. Later, the tissues were exposed to isopropanol at various concentrations (70,90 and 100\%) and dehydrated by $100 \%$ xylene. The tissue samples were then embedded in bee's wax and $5 \mu \mathrm{M}$ sections were prepared by using microtome. Then, the tissues were succeeded by hematoxylin and eosin staining and observed under microscope (magnification 40X).

\section{SEM of liver samples}

Liver tissue samples were collected $(2-4 \mathrm{~mm})$ and fixed in $2.5 \%$ glutaraldehyde for $2-6 \mathrm{~h}$ at $4^{\circ} \mathrm{C}$ for primary fixation. Then, the samples were washed with $0.1 \mathrm{M}$ phosphate buffer for $15 \mathrm{~min}$ at $4^{\circ} \mathrm{C}$. After that, $1 \%$ osmium tetroxide was used as a post fixation for $2 \mathrm{~h}$ at $4^{\circ} \mathrm{C}$. Again, the samples were washed in $0.1 \mathrm{M}$ phosphate buffer for 3 times at 15 min interval and kept at $4^{\circ} \mathrm{C}$. Later, these samples were dehydrated with acetone at various concentrations $(30,50,70,90,95$ and $100 \%)$. After that, all specimens were air dried at room temperature and critical point drying $\left(31.5^{\circ} \mathrm{C}\right.$ at $1100 \mathrm{psi})$. Finally, samples were mounted on to the aluminium stubs with adhesive tape and observed for the morphological changes using scanning electron microscope (JEOL JSM-6490LV).

\section{${ }^{1} \mathrm{H}-\mathrm{NMR}$-based serum metabolic profiling Sample preparation}

All serum samples were thawed at room temperature, $250 \mu \mathrm{l}$ of serum was taken and mixed with $250 \mu \mathrm{l}$ of $0.9 \%$ saline sodium-phosphate buffer of strength $50 \mathrm{mM}, \mathrm{pH} 7.4$ prepared in $\mathrm{D}_{2} \mathrm{O}$. Then, the samples were centrifuged at $10,000 \mathrm{rpm}$ for $5 \mathrm{~min}$ to remove any precipitates before acquiring the NMR data. A total $400 \mu \mathrm{l}$ of the supernatant sample was used in $5 \mathrm{~mm}$ NMR tubes (Wilmad Glass, USA) for data acquisition with a co-axial insert containing the known concentration of TSP (sodium salt of 3-trimethylsilyl-(2,2,3,3-d4)-propionic acid) i.e. $0.1 \%$ was used as external standard reference to aid metabolite quantification for NMR experiment. Deuterium oxide $\left(\mathrm{D}_{2} \mathrm{O}\right.$; as a co-solvent and to provide a deuterium field/frequency lock) and sodium salt of trimethylsilylpropionic acid-d $\mathrm{d}_{4}$ (TSP) used for NMR experiments, were purchased from Sigma-Aldrich (RI, USA).

\section{NMR measurements}

All NMR spectra were recorded at $298 \mathrm{~K}$ on Bruker Biospin Avance-III $800 \mathrm{MHz}$ NMR spectrometer operating at proton frequency of $800.21 \mathrm{MHz}$, equipped with CryoProbe and an actively shielded gradient unit with a maximum gradient-strength output of $53 \mathrm{G} /$ $\mathrm{cm}$. The raw NMR data were processed in Topspin-2.1 
(Bruker NMR data Processing Software). For each serum sample, one-dimensional ${ }^{1} \mathrm{H}-\mathrm{NMR}$ and diffusion edited ${ }^{1} \mathrm{H}$ NMR spectra were recorded using the Carr-Purcell-Meiboom-Gill (CPMG) pulse sequence (cpmgprld, standard Bruker pulse program) with presaturation of the water peak through irradiating it continuously during the recycle delay (RD) of $5 \mathrm{sec}$, and the bipolar pulse pair longitudinal eddy current delay (BPP-LED) sequence, respectively. Each CPMG spectrum consisted of the accumulation of 128 scans and lasted for approximately 15 minutes. To remove broad signals from triglycerides, proteins, cholesterols and phospholipids, a total spin-spin relaxation time of $60 \mathrm{~ms}(\mathrm{n}=300$ and $2 \mathrm{~T}=200 \delta \mathrm{s}$ ) with a line broadening factor of $0.3 \mathrm{~Hz}$ was applied. For diffusion edited (DE) ${ }^{1} \mathrm{H}$ NMR pulse sequence, the square gradients of $70 \%$ of the maximum gradient strength $(56 \mathrm{G} / \mathrm{cm})$ and $2 \mathrm{~ms}$ duration (followed by a delay of $200 \mu$ s to allow for the decay of eddy currents) were used. Diffusion time of $120 \mathrm{~ms}$ was used to attenuate the signals from low molecular weight compounds without affecting the lipid signals. All the spectra were processed using Topspin-2.1 (Bruker NMR data Processing Software) using standard Fourier Transformation (FT) procedure following manual phase and baseline-correction. Prior to FT, each FID was zero-filled to 4096 data points and a sine-bell apodisation function was applied. After FT, the chemical shifts were referenced internally to methyl peak of L-lactate (at $\delta=1.33 \mathrm{ppm}$ ). All recorded spectra were visually inspected for acceptability and subjected to multivariate statistical analysis to identify the altered metabolic pattern.

\section{Spectral assignment}

For unambiguous assignment of various peaks in the ${ }^{1} \mathrm{H}$ CPMG NMR spectra, two-dimensional NMR (2D NMR) spectra were acquired for selected samples including ${ }^{1} \mathrm{H}-{ }^{1} \mathrm{H}$ total correlation spectroscopy (TOCSY) and ${ }^{1} \mathrm{H}-{ }^{13} \mathrm{C}$ heteronuclear single quantum correlation (HSQC). The chemical shifts were identified and assigned as far as possible, by comparing them with the chemical shifts available with the software Chenomx 8.1 (Chenomx Inc., Edmonton, Canada). The remaining peaks in the CPMG ${ }^{1} \mathrm{H}$ NMR spectra were assigned using the existing databases and the literature reports such as The Human Metabolome Database (HMDB) [21-23].

\section{Multivariate data analysis}

NMR spectra were manually corrected for phase and baseline aberration using Topspin 2.1 (Bruker NMR data Processing Software). The CPMG ( $\delta 0.5-8.5 \mathrm{ppm})$ and diffusion edited $(\delta \quad 0.5-5.6 \mathrm{ppm})$ spectra were binned into $0.01 \mathrm{ppm}$ integrated spectral buckets using
AMIX package (Version 3.8.7, Bruker, Bio Spin). The region $(\delta$ 4.7-5.1) distorted due to water suppression were excluded from the CPMG and diffusion edited data to avoid the effects of imperfect water suppression. The data were obtained from AMIX after mean centering and normalization which was performed by dividing each data point by the sum of all data points present in the sample to compensate for the differences in concentration of metabolites among individual serum samples. The data were scaled using unit variance in which identical weight was given to all variables. The resulting data matrices were then exported into Microsoft Office Excel 2010 and used for multivariate analysis using open access web-based metabolomic data processing tool, named MetaboAnalyst [24] and Unscrambler X Software (Version 10.3, [CAMO, Oslo, Norway]). Principal component analysis was first performed on both the Carr-Purcell-Meiboom-Gill (CMPG) and diffusion edited (DE) datasets to identify the outliers. To further demonstrate the differences between the different groups, supervised partial least squares discriminate analysis (PLS-DA) was carried out to identify the metabolites significantly contributing to group differentiation. Model quality was assessed with $\mathrm{R}^{2}$ indicating the validity of models against overfitting and $\mathrm{Q}^{2}$ representing the predictive ability. Potential metabolites markers were identified from the loading plots (for PLSDA) and the scores of variable importance on projections. The statistical significance of these metabolites was calculated by t-test $(\mathrm{p}<0.05)$.

\section{Determination of $\mathrm{M} 1$ in rat plasma}

In a separate experiment, M1 was administered orally at $50 \mathrm{mg} / \mathrm{kg}$ bodyweight to albino Wistar rats $(\mathrm{n}=3)$ and blood was collected from retro orbital plexus at $0.083,0.25,0.5,1,2,4,8,12,16,24$ and 36 h. After collection, blood was centrifuged at $10,000 \mathrm{rpm}$ for $10 \mathrm{~min}$, serum was separated and kept at $-20^{\circ} \mathrm{C}$ for further HPLC determination [25,26].

\section{Preparation of plasma standards \& test samples}

A $1 \mathrm{mg} / \mathrm{ml}$ stock solution of M1 was prepared in methanol. 2, 5, 10, 25, 50, 100, 200, 250 and $500 \mathrm{ng} / \mathrm{ml}$ working solutions were prepared from the stock solution in methanol. A $100 \mu \mathrm{l}$ of blank plasma and $100 \mu \mathrm{l}$ working solutions were taken in separate tubes and vortexed for $30 \mathrm{~min}$. After that, the tubes were centrifuged at $13,000 \mathrm{rpm}$ for $5 \mathrm{~min}$ and supernatant was taken into another tube and dried at $40^{\circ} \mathrm{C}$ overnight. Later, the tubes were reconstituted with $50 \mu \mathrm{l}$ of methanol, vortexed for $10 \mathrm{~min}$ and $20 \mu \mathrm{l}$ was injected for HPLC analysis. The final concentration of working solutions were $1,2.5,5,12.5,25,50,100,125$ and $250 \mathrm{ng} / \mathrm{ml}$, respectively. Quality control (QC) samples were also 
prepared at concentrations of 3.0, 25.0 and $200.0 \mathrm{ng} /$ $\mathrm{ml}$, representing low, medium and high QC samples (LQC, MQC and HQC), respectively. Triplicate calibrants and QC samples were prepared at each concentration.

For test samples, $100 \mu \mathrm{l}$ of test plasma and $100 \mu \mathrm{l}$ methanol were taken in a test tube and vortexed for $30 \mathrm{~min}$. The other procedure was similar to previously described method.

\section{HPLC conditions}

For the determination of M1 in plasma samples, chromatographic separations were performed in a Waters 2489 HPLC (MA, USA) equipped with Spherowsorb C18, $5 \mu, 4.6 \times 250 \mathrm{~mm}$ column. The flow rate was at $1.0 \mathrm{ml} / \mathrm{min}$ and the mobile phases consisted of solvent $\mathrm{A}$ (formic acid containing water, $\mathrm{pH}$ 3.4) and solvent $\mathrm{B}$ (methanol). The elution condition was methanol:formic acid (70:30) and the total run time was $8 \mathrm{~min}$. The HPLC was run through Empower software version 2 and absorbance was measured at $230 \mathrm{~nm}$ wave length. The detector used for estimation was Waters 2489 UV-Vis detector. The final data was calculated using Winnonlin version 1.5.3 software.

\section{Statistical analysis}

Statistical analysis was carried out using GraphPad Prism 5.0 (CA, USA). All results were expressed as mean \pm standard deviation (SD). The data were analyzed by one-way analysis of variances followed by Bonferroni multiple comparison test. For biochemical estimations, statistical significance differences were considered with respect to toxic control $\left({ }^{* * *} \mathrm{p}<0.001\right.$; $\left.{ }^{* *} \mathrm{p}<0.01\right)$.

\section{Results}

\section{Characterization of $\mathrm{M} 1$}

The characterization data of our synthesized M1 as well as corresponding naturally isolated M1 is shown in the Supplementary Material. On comparing both of these, the characterization data of our synthesized M1 were found to be in good agreement with the previously published data of naturally isolated M1.

\section{Acute oral toxicity study}

No mortality and behavioral changes were observed up to 4 weeks. Various biological parameters such as ALT and AST in plasma, bilirubin and biliverdin in serum, and various biochemical parameters (SOD, CAT, PC, GSH, TBARS) in liver were measured. We found that there were no significant changes in any of these parameters following M1 treatment (dose ranging from 25 to $250 \mathrm{mg} / \mathrm{kg}$ ) as compared with normal control (Supplementary Table 1). Results obtained from acute oral toxicity studies implied that M1 was safe up to $250 \mathrm{mg} / \mathrm{kg}$ body weight dose to albino Wistar rats. Therefore, we decided to perform HCC activity in 50 and $100 \mathrm{mg} / \mathrm{kg}$ bodyweight doses.

\section{Estimation of plasma AST, ALT, biochemical} estimations \& bilirubin, biliverdin in liver Table 1 illustrates the activity of liver enzymes ALT and AST in all the experimental groups of rats. It is clear from Table 1 that both ALT and AST levels in plasma were increased significantly in toxic group (DENtreated group-II) as compared with controls (group-I). The levels of these enzymes were normalized after the oral administration of M1 and both the ALT and AST values for the M1-treated groups were similar to that of the group-I (control) rats. The levels of ALT and AST were also found to be decreased in positive control group (5-FU-treated group), but the percentage decrease after M1 therapy ( $100 \mathrm{mg} / \mathrm{kg}$ dose) is more compared with 5-FU treatment.

Table 2 shows the values for various oxidative stress parameters (SOD, CAT, GSH, TBARS and PC) in liver of all the groups. We observed that there was dramatic reduction of GSH in toxic control $(\sim 4.60 \mu \mathrm{M})$

Table 1. Effect of M1 on alanine aminotransferase and aspartate aminotransferase in plasma after oral administration of 50 and $100 \mathrm{mg} / \mathrm{kg}$ for 15 days to diethylnitrosamine-induced albino Wistar rats.

\begin{tabular}{|lll|}
\hline Groups & ALT $(\mathrm{U} / \mathrm{L})$ & AST (U/L) \\
\hline Control & $38.89 \pm 3.53$ & $26.52 \pm 4.67$ \\
\hline Toxic (DEN) & $143.50 \pm 2.70$ & $112.56 \pm 5.88$ \\
\hline DEN $+5-\mathrm{FU}$ & $68.36 \pm 5.32^{* * *}$ & $61.58 \pm 4.17^{* * *}$ \\
\hline M1 $50 \mathrm{mg} / \mathrm{kg}$ & $88.4 \pm 3.18^{* * *}$ & $47.73 \pm 1.76^{* * *}$ \\
\hline M1 $100 \mathrm{mg} / \mathrm{kg}$ & $58.04 \pm 2.84^{* * *}$ & $40.95 \pm 1.35^{* * *}$ \\
\hline $\begin{array}{l}\text { Data represented as mean } \pm \text { SD }(n=6) . \text { Statistically significant differences were observed between toxic control and test groups (one way- } \\
\text { ANOVA followed by Bonferroni multiple comparison test [*** }<0.001]) . \\
\text { ALT: Alanine aminotransferase; AST: Aspartate aminotransferase; DEN: Diethylnitrosamine. }\end{array}$ \\
\hline
\end{tabular}


Table 2. Effect of M1 on oxidative stress parameters in liver after oral administration of 50 and 100 $\mathrm{mg} / \mathrm{kg}$ for 15 days to diethylnitrosamine-induced albino Wistar rats.

\begin{tabular}{|c|c|c|c|c|c|}
\hline Groups & $\begin{array}{l}\text { SOD }(U / \mu g \text { of } \\
\text { protein) }\end{array}$ & $\begin{array}{l}\text { CAT ( } \mathrm{nM} \text { of } \mathrm{H}_{2} \mathrm{O}_{2} / \\
\mathrm{min} / \mu \mathrm{g} \text { of protein) }\end{array}$ & $\begin{array}{l}\text { Reduced GSH ( } \mu \mathrm{M} / \\
\mu \mathrm{g} \text { of protein) }\end{array}$ & $\begin{array}{l}\mathrm{PC}(\mu \mathrm{M} / \mu \mathrm{g} \text { of } \\
\text { protein) }\end{array}$ & $\begin{array}{l}\text { MDA ( } \mathrm{nM} / \mu \mathrm{g} \text { of } \\
\text { protein) }\end{array}$ \\
\hline Control & $8.34 \pm 0.57$ & $12.13 \pm 0.86$ & $8.07 \pm 0.24$ & $0.18 \pm 0.01$ & $23.15 \pm 2.69$ \\
\hline Toxic (DEN) & $2.45 \pm 0.39$ & $4.85 \pm 0.37$ & $4.60 \pm 0.27$ & $0.96 \pm 0.14$ & $98.78 \pm 4.45$ \\
\hline $\mathrm{DEN}+5-\mathrm{FU}$ & $6.99 \pm 0.43^{* * *}$ & $9.30 \pm 0.35^{* * *}$ & $7.30 \pm 0.39^{* * *}$ & $0.35 \pm 0.07^{* * *}$ & $46.24 \pm 0.26^{* * *}$ \\
\hline M1 $50 \mathrm{mg} / \mathrm{kg}$ & $5.92 \pm 0.72^{* * *}$ & $6.51 \pm 0.29^{* \star \star}$ & $5.40 \pm 0.42^{* *}$ & $0.46 \pm 0.08^{\star * *}$ & $51.60 \pm 1.009^{* * *}$ \\
\hline M1 $100 \mathrm{mg} / \mathrm{kg}$ & $6.14 \pm 0.17^{* * *}$ & $7.52 \pm 0.7^{\star \star *}$ & $6.69 \pm 0.30^{* * *}$ & $0.40 \pm 0.02^{* * *}$ & $45.20 \pm 1.05^{\star \star *}$ \\
\hline
\end{tabular}

than normal control $(\sim 8.07 \mu \mathrm{M})$. Improvement in GSH level was observed after M1 treatment $(\sim 5.40 \mu \mathrm{M}$ for $50 \mathrm{mg} / \mathrm{kg}$ and $\sim 6.69 \mu \mathrm{M}$ for $100 \mathrm{mg} / \mathrm{kg}$ ). Similar trend was observed for SOD, where we found that SOD level decreased to $20-30 \%$ in toxic control as compared with normal group. This level was again improved up to $70-80 \%$ in M1-treated groups. The CAT enzyme activity was found to be improved for both positive control and treated groups than toxic control (Table 2). Further the tissue malondialdehyde (MDA) and PC formation was also measured to evaluate the protective action of M1. The MDA formation was approximately $98 \mathrm{nM}$ for toxic control which was reduced after $\mathrm{M} 1$ therapy $(\sim 50 \mathrm{nM})$. Also, the PC formation was higher for toxic control $(\sim 0.96 \mu \mathrm{M})$ which was reduced to approximately half for M1-treated rats $(\sim 0.40 \mu \mathrm{M}$; Table 2$)$. The positive control group (treated with 5-FU) also exhibited significant increase in SOD, CAT, GSH levels and significantly decreased in PC and MDA levels.

Both conjugated bilirubin and biliverdin levels in liver were increased in toxic groups ( $60 \mathrm{ng})$. As shown in Table 3, bilirubin level was decreased two times ( $\sim 35 \mathrm{ng}$ ) in both 5-FU and M1-treated rats. Similar trend was observed for biliverdin assay where we found that biliverdin concentration was decreased for M1-treated rats ( 16 ng) than toxic control ( 28 ng).

\section{Histopathology and SEM analysis of liver}

The histopathology of the liver tissue was examined under a light microscope and presented in Figure 1. Group I expressed (Figure 1A) the normal architecture of the liver cells with normal nucleus $(\mathrm{N})$ and Kupffer's cells (K). DEN Group-II (Figure 1B) depicted the degeneration of nucleus containing nucleolus $(\mathrm{dN})$ and Kupffer's cells $(\mathrm{K})$ along with the ruptured hepatic cells (RC), tumor cells (TC) and tumor anaplastic cells (TA). Group III (Figure 1C) rats showed the normal histological appearance of liver cells ( $\&$ K). In Groups IV and $\mathrm{V}$ (Figure 1D \& E), it was observed that the damaged liver architecture was altered, necrosis healed and the cellular degeneration was found to be lower depending upon the dose. SEM analysis expressed the similar trends where lesions were less prominent in M1-treated rats as compared with DEN group (Supplementary Figure 3).

\section{${ }^{1} \mathrm{H}$-NMR method for serum metabolites profiling}

Typical ${ }^{1} \mathrm{H}$ CPMG NMR spectra of serum samples obtained from different groups are shown in Supplementary Figure 4. The NMR spectra showed

Table 3. Effect of M1 on bilirubin and biliverdin in liver after oral administration of 50 and $100 \mathrm{mg} /$ $\mathrm{kg}$ for 15 days to diethylnitrosamine-induced albino Wistar rats.

\begin{tabular}{|c|c|c|}
\hline Groups & Bilirubin ( $\mathrm{ng} / \mu \mathrm{g}$ of protein) & Biliverdin ( $\mathrm{ng} / \mu \mathrm{g}$ of protein) \\
\hline Control & $25.80 \pm 2.70$ & $15.11 \pm 2.40$ \\
\hline DEN & $60.48 \pm 3.55$ & $28.62 \pm 3.77$ \\
\hline $\mathrm{DEN}+5-\mathrm{FU}$ & $34.70 \pm 2.12^{* * *}$ & $18.39 \pm 3.11^{* * *}$ \\
\hline M1 $50 \mathrm{mg} / \mathrm{kg}$ & $50.59 \pm 1.39^{* * *}$ & $21.19 \pm 2.42^{* *}$ \\
\hline M1 $100 \mathrm{mg} / \mathrm{kg}$ & $40.64 \pm 3.47^{* * *}$ & $16.95 \pm 1.96^{\star \star *}$ \\
\hline
\end{tabular}



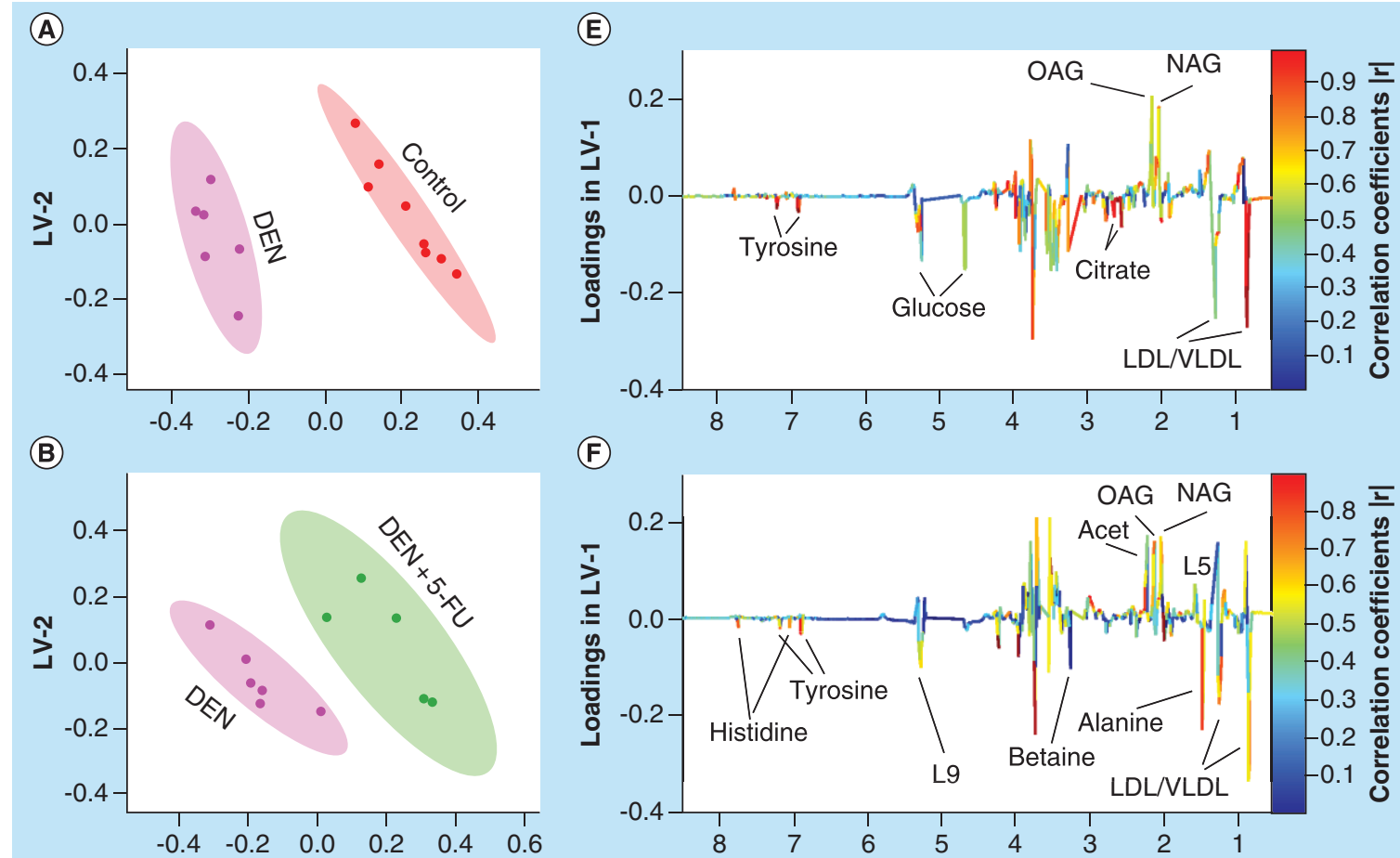

(C)

(F)

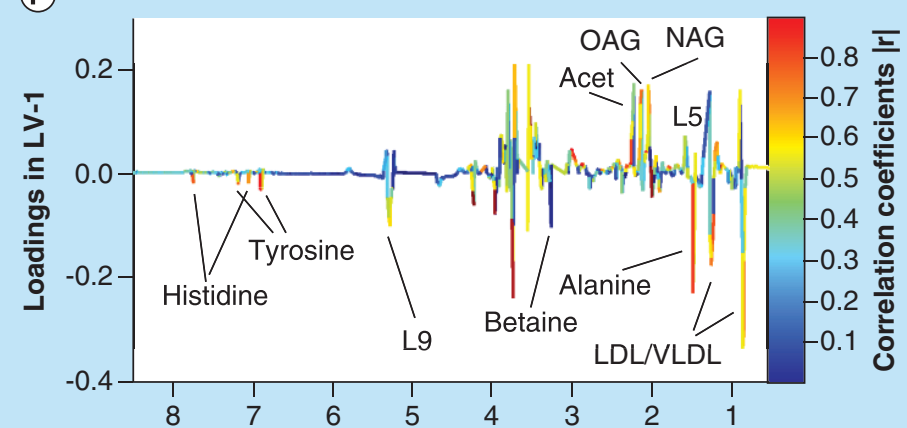

(G)
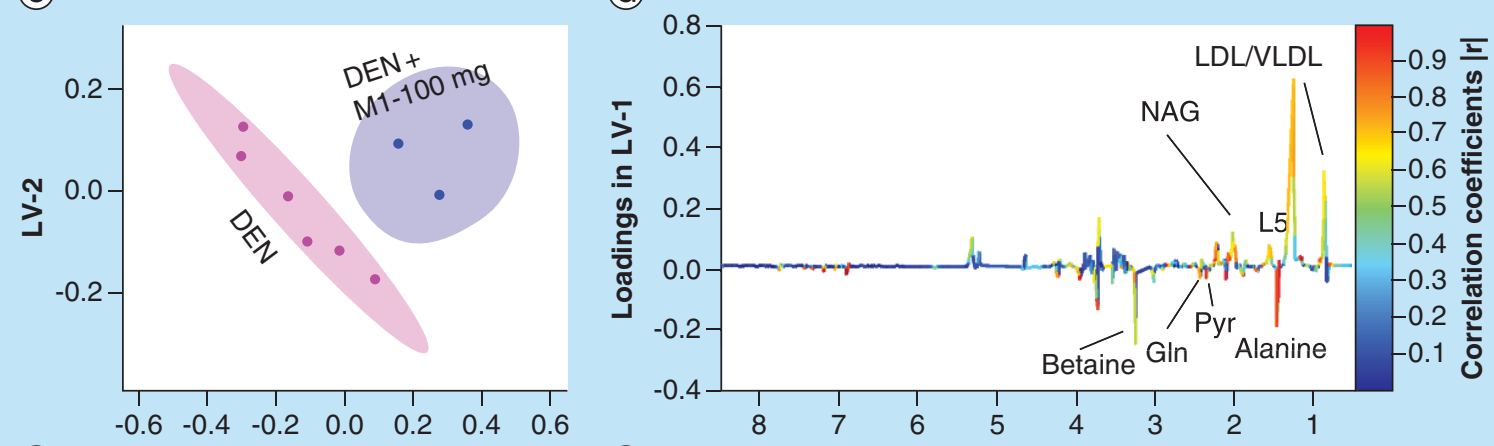

(D)

(H)
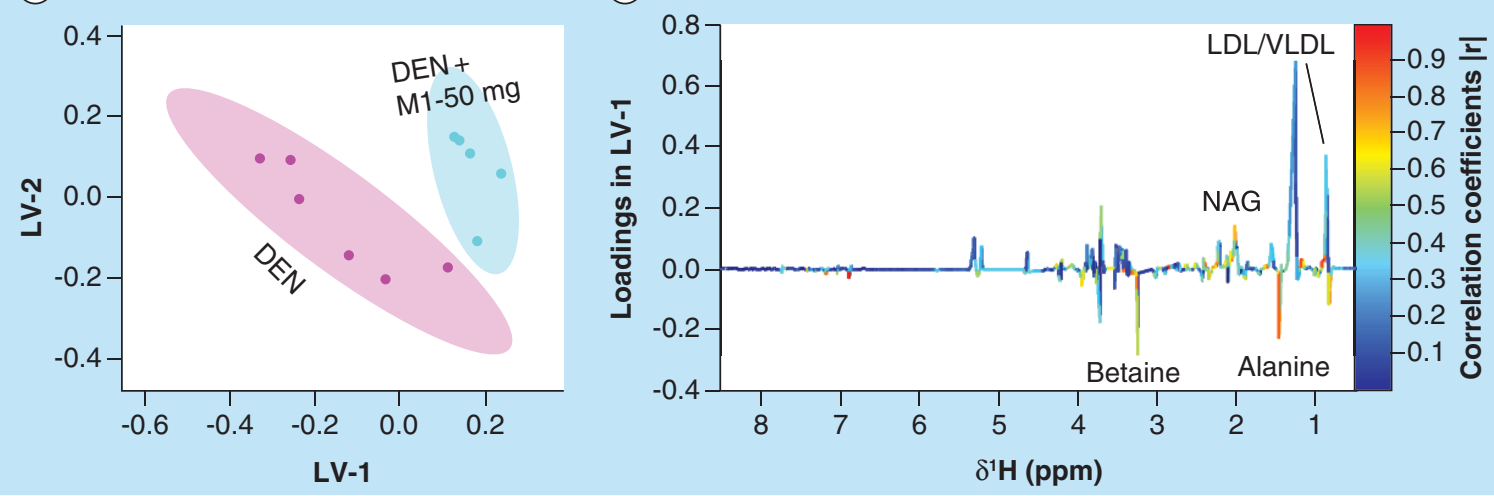

Figure 2. Partial least squares discriminate analysis score plots. The plots are derived from $1 D$ CPMG ${ }^{1} H$ NMR spectra of rat serum samples between (A) control \& DEN, (B) DEN \& DEN+5-FU, (C) DEN \& DEN+M1-100 mg and (D) DEN \& DEN+M1-50 mg. (E, F, G \& H) Shows the color coded coefficient loading plot corresponding to the PLSDA analysis shown in (A, B, C \& D), respectively. The loading plots clearly demonstrate the metabolites responsible for the discrimination of the two groups in the corresponding score plots. Peaks in the positive direction $(>0)$ indicate the metabolites which are more abundant in the groups in the positive direction of first principal component. Consequently, metabolites which are more abundant in the groups in the negative direction $(<0)$ of first primary component are presented as peaks in the negative direction.

DEN: Diethylnitrosamine; LDL: Low-density lipoprotein; NAG: N-acetyl glycoprotein; OAG: O-acetyl glycoprotein; PLS-DA: Partial least squares discriminate analysis; VLDL: Very LDL. 

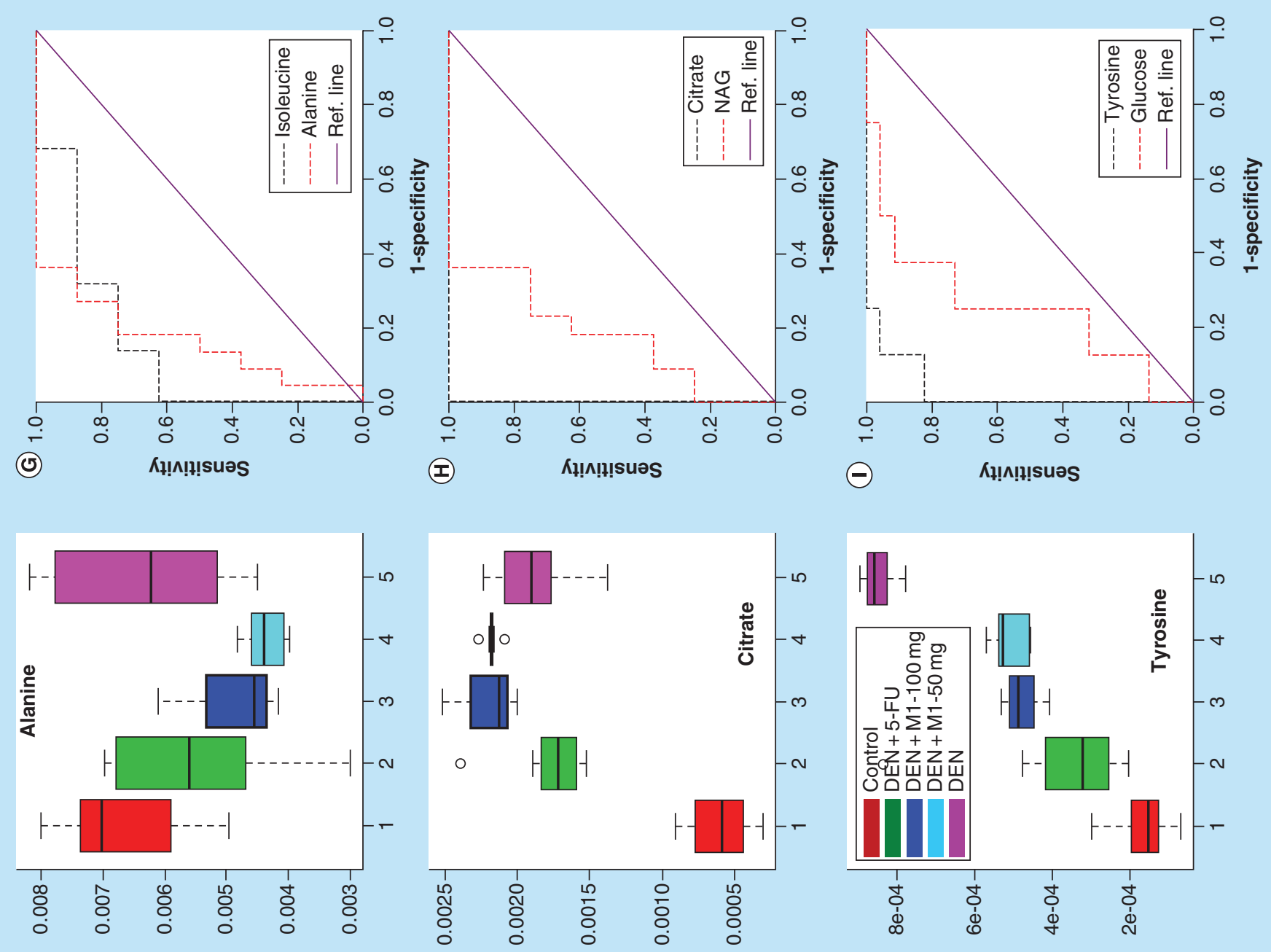

(a)

(4)

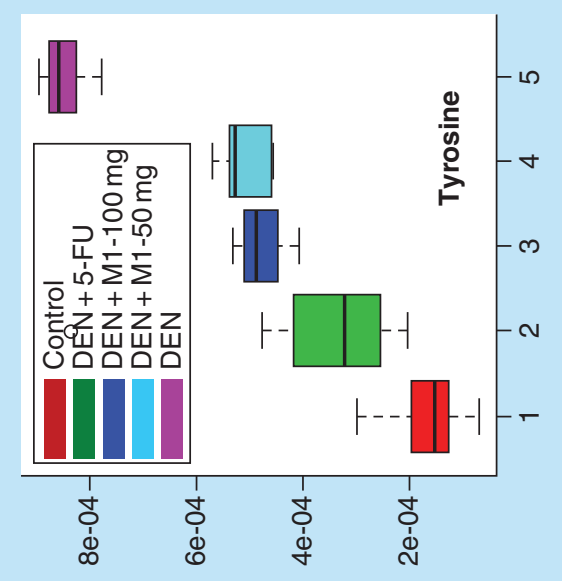

(4)
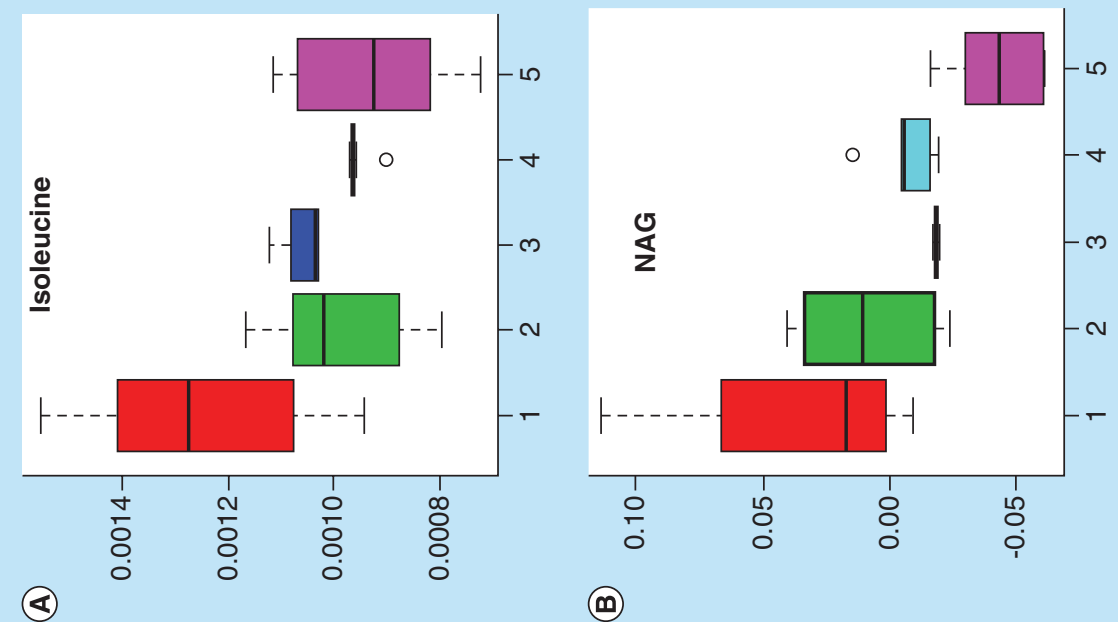

(0)

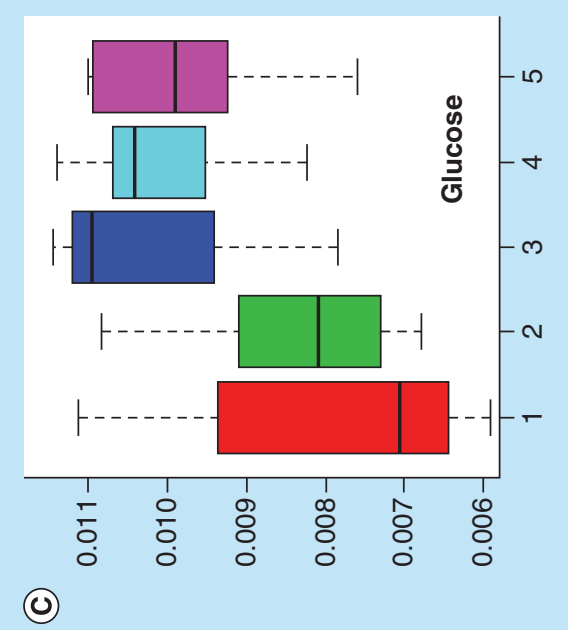


Figure 3. Representative box-cum-whisker plots of the normalized integral areas of low molecular weight metabolites (cont. from previous page). The plots are drawn from CPMG spectra (A) isoleucine, (B) NAG, (C) glucose, (D) alanine, (E) citrate and (F) tyrosine of control, DEN, DEN+5-FU, DEN+M1-100 mg and DEN+M1-50 mg rat sera. In the box plots, the boxes denote interquartile ranges, horizontal lines inside the box denote that the median, and bottom and top boundaries of boxes are 25th and 75th percentiles, respectively. Lower and upper whiskers are 5th and 95th percentiles, respectively. The corresponding ROC curves for these metabolites have also been displayed in (G-I), respectively.

DEN: Diethylnitrosamine; NAG: N-acetyl glycoprotein; ROC: Receiver's operating characteristic.

signals mainly from lipids/lipoproteins (e.g., lowdensity lipoprotein $[\mathrm{LDL}]$, very low density lipoprotein [VLDL], unsaturated fatty acids, etc., and amino acids (e.g., alanine, valine, lysine, leucine, isoleucine, histidine, tyrosine, glutamine, glutamate and proline, among others). Other identified metabolites were glucose, choline, creatine, acetoacetate, acetone, acetate, citrate, lactate, $N$-acetyl and $O$-acetyl glycoproteins (NAG, OAG). We performed the multivariate data analysis to find out the HCC-induced metabolic alterations and further to reveal the effect of M1 treatment on these metabolic profile modulations.

\section{Metabolic changes in response to HCC in rats}

The principal component analysis score plot shown in Supplementary Figure 5 exhibited clear trend of clustering in different groups and no outlier sample was detected. To obtain satisfactory classification and select metabolite markers, pairwise PLS-DA analysis was further performed on NMR data matrices. The combined PLS-DA score plot for all groups (Supplementary Figure 6) and pairwise PLS-DA score plots (Figure 2A) showed that the clusters of DEN-treated rats are well separated from normal control group with a significantly higher quality of fit and predictability $\left(\mathrm{R}^{2}=0.99, \mathrm{Q}^{2}=0.95\right.$; Table 4$)$, indicating that significant metabolic changes were induced by DEN treatment. The corresponding loading plot in the first latent variable of PLS-DA is shown in Figure 2E which clearly revealed the metabolites responsible for discrimination of the two groups. The loading plots were color-coded according to the absolute value of correlation coefficients $(|\mathrm{r}|)$, where a hot-colored signal (red) indicated more significant contribution to class separation than a cold-colored one (blue). The key observed metabolic differences between the control and DEN group along with their chemical shifts, variable importance on projection score and p-value are listed in Table 5. Compared with control group, DEN-treated rats had significant elevation of lipids, lipoproteins (LDL), glucose, creatine, citrate, pyruvate, tyrosine and choline, together with decreased level for amino acids (such as leucine, isoleucine and glutamate), acetate, acetoacetate and NAG and OAG (Figures 3, 4 \& Table 5).

\section{Metabolic effects of M1 \& 5-FU treatment}

The combined PLS-DA score plot of all the five groups (Supplementary Figure 6) showed that 5-FU and M1 treatments shifted the state from DEN-treated group to back toward the control group. The pairwise score plots between M1, 5-FU-treated groups and DEN-treated group are shown in Figure 2B-D along with their loading plots (Figure $2 \mathrm{~F}-\mathrm{H}$ ) which clearly revealed that the treatment groups are well separated from DEN-treated group. The significantly high values of $\mathrm{R}^{2}$ and $\mathrm{Q}^{2}(>0.5$ as enlisted in Table 4) infer that all the PLS-DA models possessed a satisfactory fit with good predictive power. Further, we found that the metabolic alterations which were observed in DEN-treated group get ameliorated after the M1 treatment. For example, the metabolites which were increased in DEN-treated group such as lipids, LDL, glucose, pyruvate, choline, lysine and tyrosine get decreased in the M1-treated groups. Similarly, the levels of amino acids, NAG, OAG and acetoacetate were increased after M1 treatment. Similar amelioration of the metabolites was also observed after 5-FU treatment in HCC rats, that is, the metabolites which were increased in HCC rats get decreased after the 5-FU treatment and vice versa.

\section{Determination of plasma concentration of M1 using HPLC}

A linear regression performed over a range of $1-250 \mathrm{ng} / \mathrm{ml}$ yielded a correlation coefficient $\left(\mathrm{r}^{2}\right)$ of $>0.9$ for M1 (Table 6). The accuracy of the assay was found to be within $81-92 \%$ and recovery of the samples was $67-83 \%$. The retention time for M1 was 5.24 min (Figure 5A). As depicted in Table 6 and Figure $5 \mathrm{~B}$, the maximum plasma concentration $\left(\mathrm{C}_{\max }\right)$ and time required to reach the maximum concentration in plasma $\left(\mathrm{T}_{\max }\right)$ were $178.4 \mathrm{ng} / \mathrm{mL}$ and $4 \mathrm{~h}$, respectively. The plasma concentration reached to $50 \%\left(\mathrm{t}_{1 / 2}\right)$ at $6.05 \mathrm{~h}$. The total area under the curve after $36 \mathrm{~h}$ was $2065.05 \mathrm{ng} . \mathrm{h} / \mathrm{mL}$.

\section{Discussion}

HCC is the third most frequent cause of cancer deaths in both developed and developing countries. The clinical outcome of HCC treatment remains unsatisfactory due to its resistance toward the presently usable chemotherapeutic agents. Furthermore, the usage of synthetic antiproliferative chemotherapeutic agents has declined due to their potential toxicity to human body. It is, therefore, necessary to investigate potential therapeutic agents for the clinical benefits among cancerous patients. Natural 

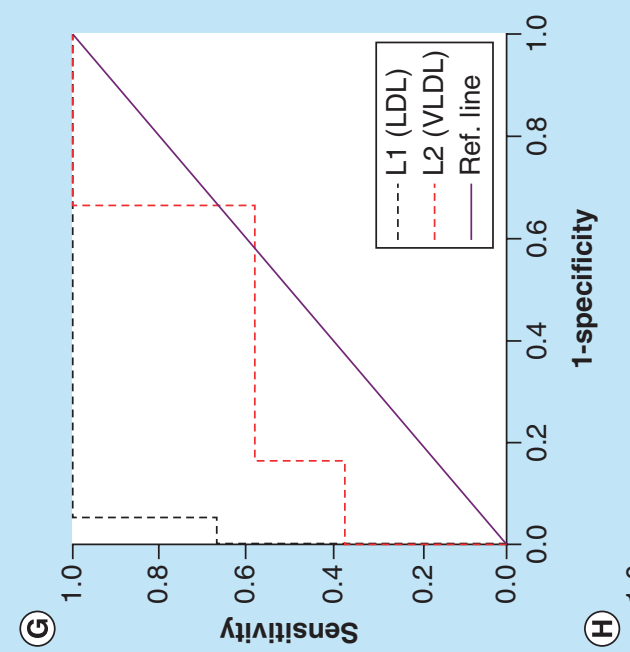

(I)

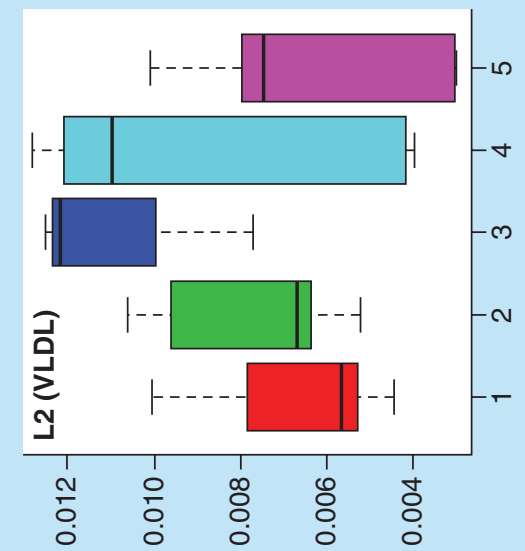

(9)

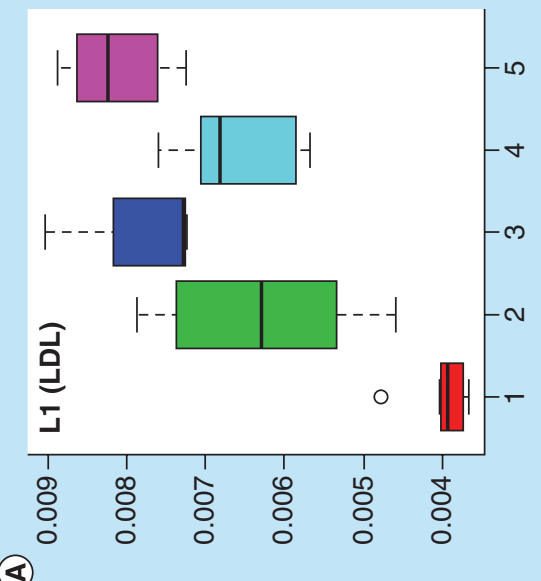

(a)

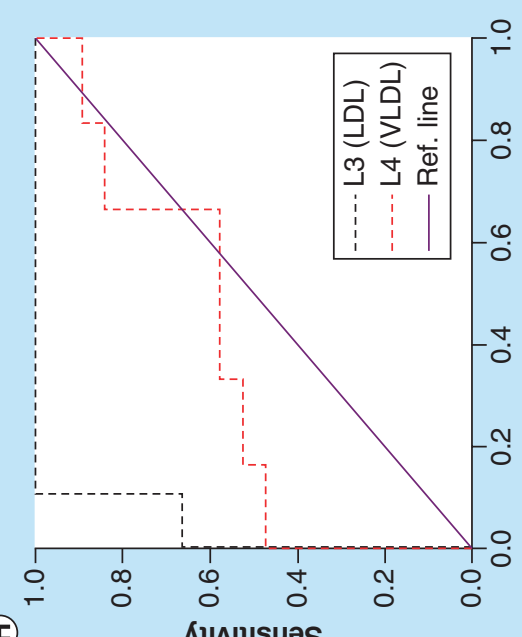

K!!ก!!! suəS
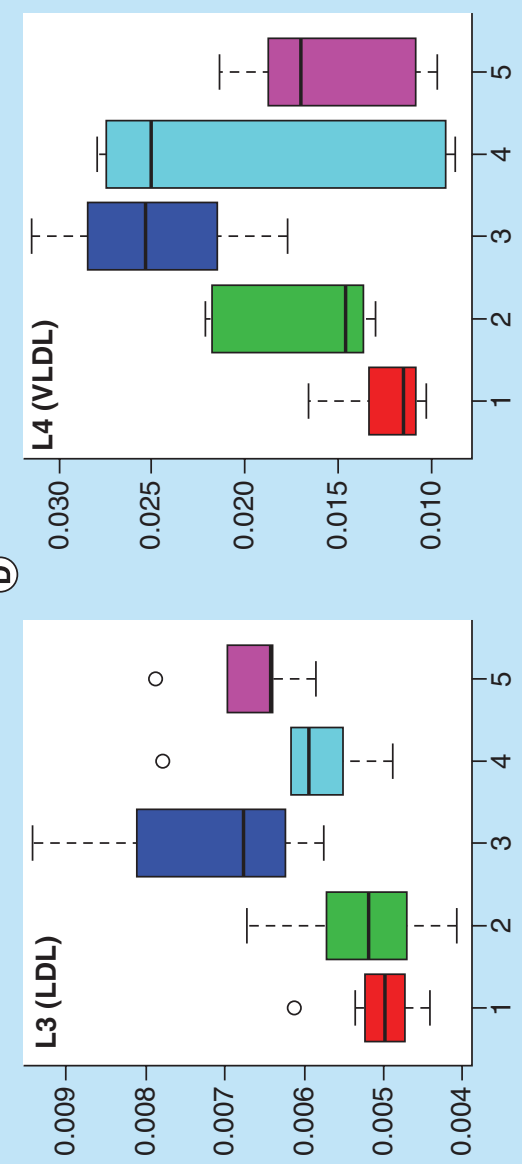

(0)
(4)
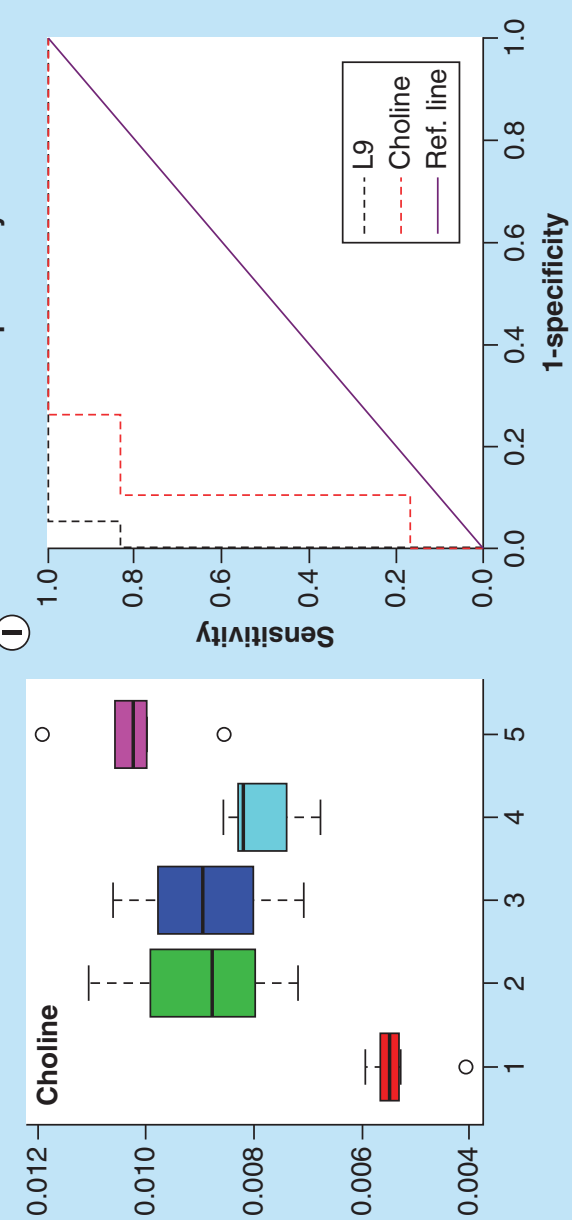

(4)

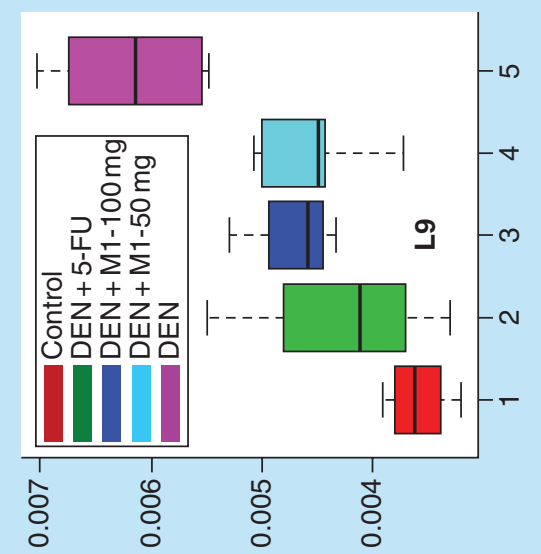


Figure 4. Quantitative variations of low-density lipoprotein, very low-density lipoprotein, lipids and choline in control, diethylnitrosamine, DEN+5-FU, DEN+M1-100 mg and DEN+M1-50 mg rat sera represented as box-cumwhisker plots of normalized integral area (cont. from previous page). Horizontal line inside the box is the median and bottom and top boundaries of boxes are 25th and 75th percentiles, respectively. Lower and upper whiskers are 5th and 95th percentiles, respectively. The corresponding ROC curves for these metabolites have also been displayed in (G-I), respectively.

DEN: Diethylnitrosamine; LDL: Low-density lipoprotein; ROC: Receiver's operating characteristic; VLDL: Very LDL.

products are a good source from which to develop new medications for disease treatment as the compounds obtained from natural origin are often safe and less toxic in nature [3]. During the search for new HCC therapeutic agents, our group recently isolated M1 from MP seeds, which had good antiproliferative action on Huh-7 cells [12]. In this study, we investigated the in vivo antiproliferative action of $\mathrm{M} 1$ via its oral administration to hepatocarcinogenic albino Wistar rats. Results presented in the current study demonstrated the protective effect of $\mathrm{M} 1$ against DEN-induced HCC and are discussed in details further.

The levels of serum transaminases (AST and ALT) were found to be increased in DEN-treated rats as compared with the control group I indicating the hepatic damage in DEN group [16]. The enhanced levels of AST and ALT were lowered after the treatment significantly and dose-dependently with M1 inferring its ability to reduce hepatocarcinogenic features in HCC-bearing rats, similar to 5-FU. Various antioxidant parameters such as GSH, CAT and SOD were decreased significantly with simultaneous PC and MDA increased in DEN-treated rats, compared with that of control group. The alterations of these parameters reflect a good correlation of transformed cells in cancerous condition [27]. The levels of GSH, CAT and SOD were restored back to normalcy upon treatment with M1 in dose-dependent manner and in positive control group. Also the levels of MDA and PC were decreased significantly in M1 and 5-FU-treated groups, when compared with DEN rats.
GSH is a tripeptide which is most abundant for all tissues including liver and plays major role in oxidationreduction process to scavenge free radicals during oxidative damage [28]. M1-treated groups showed increase in GSH levels in dose-dependent manner and in 5-FU group bringing nearby to normal levels. Improvement in GSH levels after treatment revealed that M1 might be effective against DEN-induced liver damage. Further, the antioxidative enzymes SOD and CAT in liver provide protective defense against the reactive oxygen species. The enzyme CAT catalyzes the conversion of $\mathrm{H}_{2} \mathrm{O}_{2}$ to oxygen and water, thereby providing protection against reactive oxygen species and SOD neutralizes superoxide free radical in normal physiological situations [29]. These enzymes' action is reduced due to the decreased expression of these antioxidants in DENinduced HCC [30,31]. The restoration of the activities of SOD and CAT toward normalcy after the administration of M1 could be due to the antioxidant potency of M1 to scavenge reactive oxygen species.

Oxidation of lipids is an important parameter to measure oxidative stress-induced liver damage during cancerous condition [32]. The assessment of the degree of lipid peroxidation can be obtained by the measurement of MDA, which is one of the end products of lipid peroxidation. In the present study, oxidative stress leads to fourfold increase in the levels of MDA in DEN-treated rats. The decrease in the levels of MDA after treatment with M1 and 5-FU could be due to scavenging of the reactive free radi-

\begin{tabular}{|c|c|c|c|c|c|}
\hline Comparison & NMR spectra & $\mathbf{R}^{2} \mathbf{X}($ cum $)$ & $R^{2} Y($ cum $)$ & $\mathrm{Q}^{2}$ (cum) & $\begin{array}{l}\text { Number of latent } \\
\text { variables }\end{array}$ \\
\hline Control vs DEN & 1D CPMG & 0.79 & 0.99 & 0.95 & 3 \\
\hline DEN vs DEN+5-FU & 1D CPMG & 0.66 & 0.78 & 0.52 & 2 \\
\hline DEN vs DEN+M1-100 mg & 1D CPMG & 0.79 & 0.97 & 0.54 & 3 \\
\hline DEN vs DEN+M1-50 mg & 1D CPMG & 0.74 & 0.95 & 0.53 & 3 \\
\hline Control vs DEN & 1D diffusion edited & 0.66 & 0.96 & 0.90 & 2 \\
\hline DEN vs DEN+5-FU & 1D diffusion edited & 0.89 & 0.99 & 0.89 & 4 \\
\hline DEN vs DEN+M1-100 mg & 1D diffusion edited & 0.66 & 0.84 & 0.59 & 2 \\
\hline DEN vs DEN+M1-50 mg & 1D diffusion edited & 0.92 & 0.95 & 0.55 & 4 \\
\hline
\end{tabular}


Table 5. Key observed metabolic differences between the healthy control and diethylnitrosamine induced rat after M1 treatment.

\begin{tabular}{|c|c|c|c|c|c|c|c|}
\hline \multirow[t]{2}{*}{ Metabolites } & \multirow[t]{2}{*}{ Chemical shift } & \multirow{2}{*}{$\begin{array}{l}\text { Variation in DEN with } \\
\text { respect to control }\end{array}$} & \multirow[t]{2}{*}{ VIP } & \multirow[t]{2}{*}{ p-value } & \multicolumn{3}{|c|}{ Variation with respect to DEN } \\
\hline & & & & & 5-FU & M1-100mg & M1-50 mg \\
\hline Leu & 0.96 & $\downarrow$ & 2.01 & 0.001 & - & $\uparrow$ & $\uparrow$ \\
\hline ILeu & 1.00 & $\downarrow$ & 1.17 & 0.008 & - & $\uparrow$ & - \\
\hline Ala & 1.48 & - & - & - & $\downarrow$ & $\downarrow$ & $\downarrow$ \\
\hline Lys & 1.89 & $\uparrow$ & 1.86 & 0.002 & $\downarrow$ & $\downarrow$ & $\downarrow$ \\
\hline Ace & 1.92 & $\downarrow$ & 1.79 & 0.04 & $\downarrow$ & $\downarrow$ & $\downarrow$ \\
\hline NAG & 2.02 & $\downarrow$ & 3.76 & 0.003 & $\uparrow$ & $\uparrow$ & $\uparrow$ \\
\hline OAG & 2.13 & $\downarrow$ & 3.46 & 0.03 & $\uparrow$ & - & $\uparrow$ \\
\hline Acet & 2.23 & - & - & - & $\uparrow$ & $\uparrow$ & $\uparrow$ \\
\hline Acac & 2.28 & $\downarrow$ & 1.56 & $<0.001$ & $\uparrow$ & $\uparrow$ & $\uparrow$ \\
\hline Glu & 2.35 & $\downarrow$ & 1.09 & 0.02 & - & - & - \\
\hline Pyr & 2.37 & $\uparrow$ & 1.21 & 0.045 & $\downarrow$ & $\downarrow$ & $\downarrow$ \\
\hline Cit & 2.52 & $\uparrow$ & 1.71 & $<0.001$ & - & $\uparrow$ & $\uparrow$ \\
\hline $\mathrm{Cr}$ & 3.03 & $\uparrow$ & 1.64 & 0.002 & $\uparrow$ & $\downarrow$ & $\uparrow$ \\
\hline Chol & 3.22 & $\uparrow$ & 4.19 & $<0.001$ & $\downarrow$ & $\downarrow$ & $\downarrow$ \\
\hline Betaine & 3.265 & - & - & - & $\downarrow$ & $\downarrow$ & $\downarrow$ \\
\hline Glucose & $\begin{array}{l}3.23-3.92,4.63 \\
5.21\end{array}$ & $\uparrow$ & $2.69-1.00$ & 0.05 & $\downarrow$ & - & - \\
\hline Tyr & 6.89 & $\uparrow$ & 2.05 & $<0.001$ & $\downarrow$ & $\downarrow$ & $\downarrow$ \\
\hline L1 (LDL) & $0.83-0.87$ & $\uparrow$ & 5.6 & $<0.001$ & $\downarrow$ & $\downarrow$ & $\downarrow$ \\
\hline L2 (VLDL) & $0.87-0.90$ & - & - & - & $\uparrow$ & $\uparrow$ & $\uparrow$ \\
\hline L3 (LDL) & $1.23-1.27$ & $\uparrow$ & 3.02 & $<0.001$ & $\downarrow$ & - & $\downarrow$ \\
\hline L4 (VLDL) & $1.27-1.30$ & - & - & - & $\uparrow$ & $\uparrow$ & $\uparrow$ \\
\hline L6 & 2.00 & $\uparrow$ & 2.43 & $<0.001$ & $\downarrow$ & - & $\downarrow$ \\
\hline L8 & 2.74 & $\uparrow$ & 2.05 & $<0.001$ & $\downarrow$ & - & - \\
\hline L9 & 5.28 & $\uparrow$ & 2.65 & 0.001 & $\downarrow$ & - & $\downarrow$ \\
\hline
\end{tabular}

cals involved in the peroxidation and, hence, may result in the inhibition of lipid peroxidation. Further, the carboxyl group of protein becomes oxidized due to formation of reactive oxygen species [33] and converted to $\mathrm{PC}$ which is also an important marker for oxidative stress-induced damage in cancerous cell. As depicted in the present study, PC levels were highly decreased in M1-treated rats, which again reveals the protective action M1 against DEN-induced hepatocellular damage. The upregulation of bilirubin and biliverdin, two unconjugated pigment metabolites, was also observed in DEN-treated rats, indicating the liver cells damage during HCC [34]. The levels of both the unconjugated pigment were restored to normal value after the treatment with M1 suggesting its protective action.

Further evidence of protective action was observed through histopathology and SEM analysis. Both analyses showed that the DEN-treated animals had irregular shaped nuclei with irregular cytoplasm, which might be due to excessive free radical generation during DEN administration [35]. M1 and 5-FU-treated groups demonstrated less ruptured and denatured cells (RC and DC) than toxic control, signifying their protective action against HCC. Similar trends were observed during SEM analysis.

NMR-based serum metabolomics coupled with multivariate statistical analysis was further carried out to 


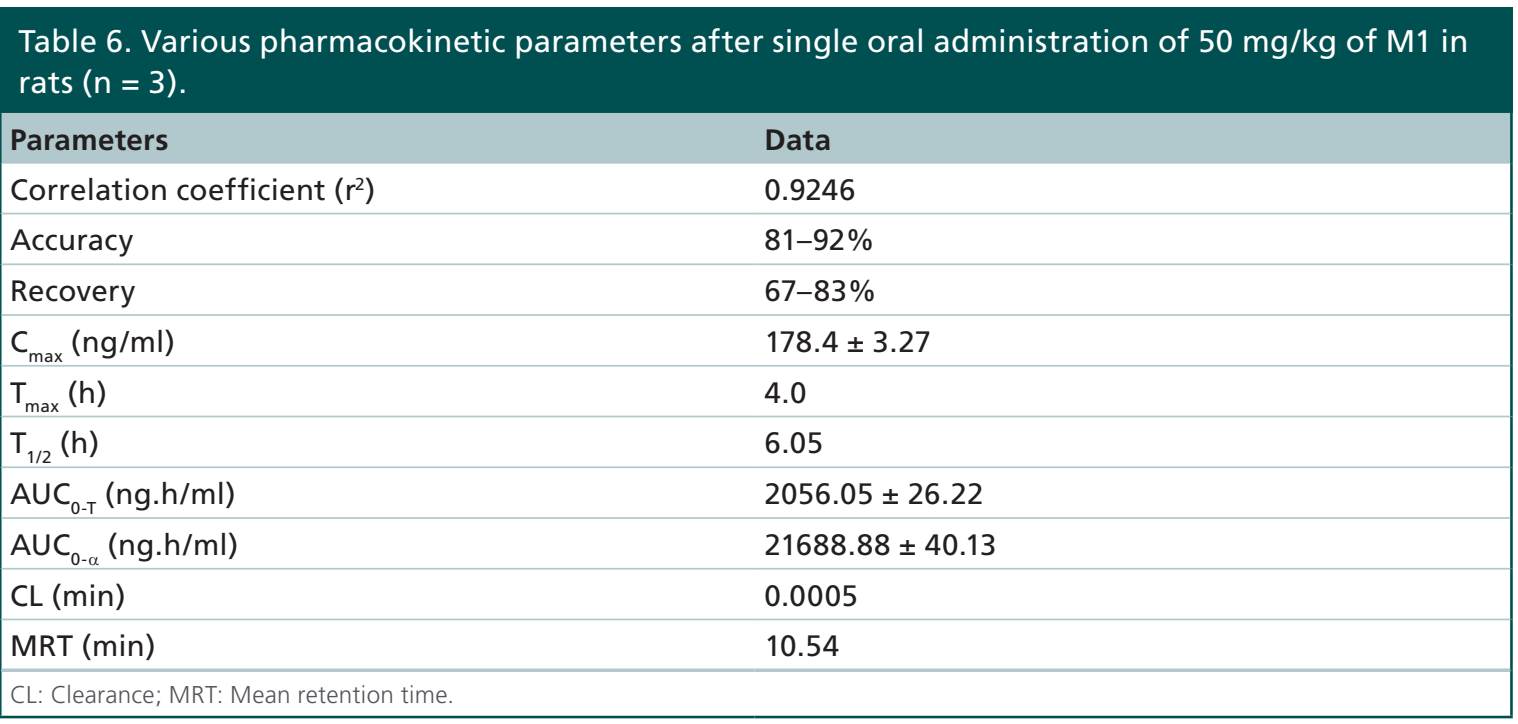

investigate the HCC-induced metabolic alterations and further to evaluate the effect of M1 treatment on these alterations. A number of metabolites were identified that differed between DEN-treated group and normal control group. We found that HCC rat sera have higher levels of lipids, LDL lipoprotein, glucose, tyrosine, citrate, choline, pyruvate, creatine and lower levels of amino acids (such as leucine, isoleucine and glutamate), $N$ - and $O$-acetyl glycoproteins, acetate and acetoacetate. The biological pathways involved in the metabolism of these metabolites and their biological roles were determined by enrichment analysis using MetaboAnalyst [36]. The metabolic pathways play a crucial role in the development of HCC that include the metabolism of glycine, serine and threonine, valine, leucine, isoleucine, pyruvate, phenylalanine, tyrosine, tryptophan, tyrosine and glyoxylate.

We observed significant increase in the levels of LDL, lipids and choline in HCC rats compared with normal rats. LDL mainly deliver cholesterol to cells, where it is used in membranes. Downregulated expression of LDL receptors in liver cells leads to elevation in levels of circulated LDL as it is not cleared from circulation due to the low functioning of LDL receptors [37]. Further fatty acids and lipids are also used for energy production such as $\beta$-oxidation, therefore, the increase in their level could be due to the results of energy requirement for cell membrane synthesis and fast-growing proliferation [38], and these alterations in lipogenesis might be an important factor for tumor development and growth. The decreased level of acetate, which is the end product of lipid metabolism, also reflects a disturbed lipid metabolism in HCC rats. Choline is an important intermediate of phospholipid metabolism which is an essential component in membrane structure and inflammatory mediators. High levels of choline could be due to the induced membrane transport of choline, upregulation of phospholipases and upregulation of choline kinase activity [39]. Choline is also known to attenuate immune inflammation through a cholinergic anti-inflammatory pathway; [40] therefore; the elevated choline level may be due to the activation of choline metabolism to dampen the inflammation associated with hepatic injury in HCC.

Significantly altered energy metabolism was observed in the DEN-treated rats. The citric acid levels was found to be elevated in the HCC rats suggesting an altered tricarboxylic acid (TCA) cycle, which is in agreement with the commonly observed mitochondrial dysfunction in cancer [41]. The alterations in the citrate acid levels demonstrate the high energy demand as well as the altered enzyme activities in association with tumor growth. The decrease in the levels of acetoacetate in HCC rats also suggests the impairment in the TCA cycle and energy metabolism in liver mitochondria. The depleted levels of leucine and isoleucine are in concordance with the previous study on human HCC [42] which was reported to be associated with the reduced translation from succinyl-CoA because of the impaired TCA cycle as discussed above. Elevated levels of pyruvate were also found in the sera of HCC rats as compared with normal, which could be due to higher energy consumption or due to an increase in anaerobic cell respiration [42]. Serum creatine, which is a key intermediate in energy metabolism, was significantly elevated in HCC rats compared with normal rats and might be associated with increase in energy demand due to tumor growth. The levels of glucose was found to be higher in HCC rats compared with normal controls, whereas that of lactate, the end point product of the glycolysis pathway, was decreased (but not significant), suggesting dampened glycolysis in DEN-treated rats. Glutamine, lysine, and tyrosine, were also increased in 
the HCC rats which might be due to increased catabolism $[43,44]$. Taken together, due to aberrantly higher rates of cell proliferation, the rates of aerobic glycolysis, fatty acid synthesis and TCA cycle were higher in HCC rats to keep up with high energy and biomass demands as well as the altered enzyme activities in association with tumor growth.

The effect of M1 and 5-FU treatment on the metabolic alterations due to HCC as discussed above was investigated further. We found that the M1 treatment of DEN rats leads to the ameliorations of the HCC- induced metabolic alterations, signifying its antiproliferative properties. Similar ameliorations of the HCCinduced metabolic alterations were also observed in the standard 5-FU-treated group inferring that M1 treatment is equipotent to the standard 5-FU in restoration of altered metabolic patterns. Under the M1 treatment, metabolic markers (leucine, isoleucine, lysine, acetoacetate, pyruvate, creatine, choline, tyrosine, LDL lipoproteins and fatty acids) were modulated toward the level of normal controls. $N$-acetyl glycoproteins, an acute phase protein [45], have been found to be increased after

(A)

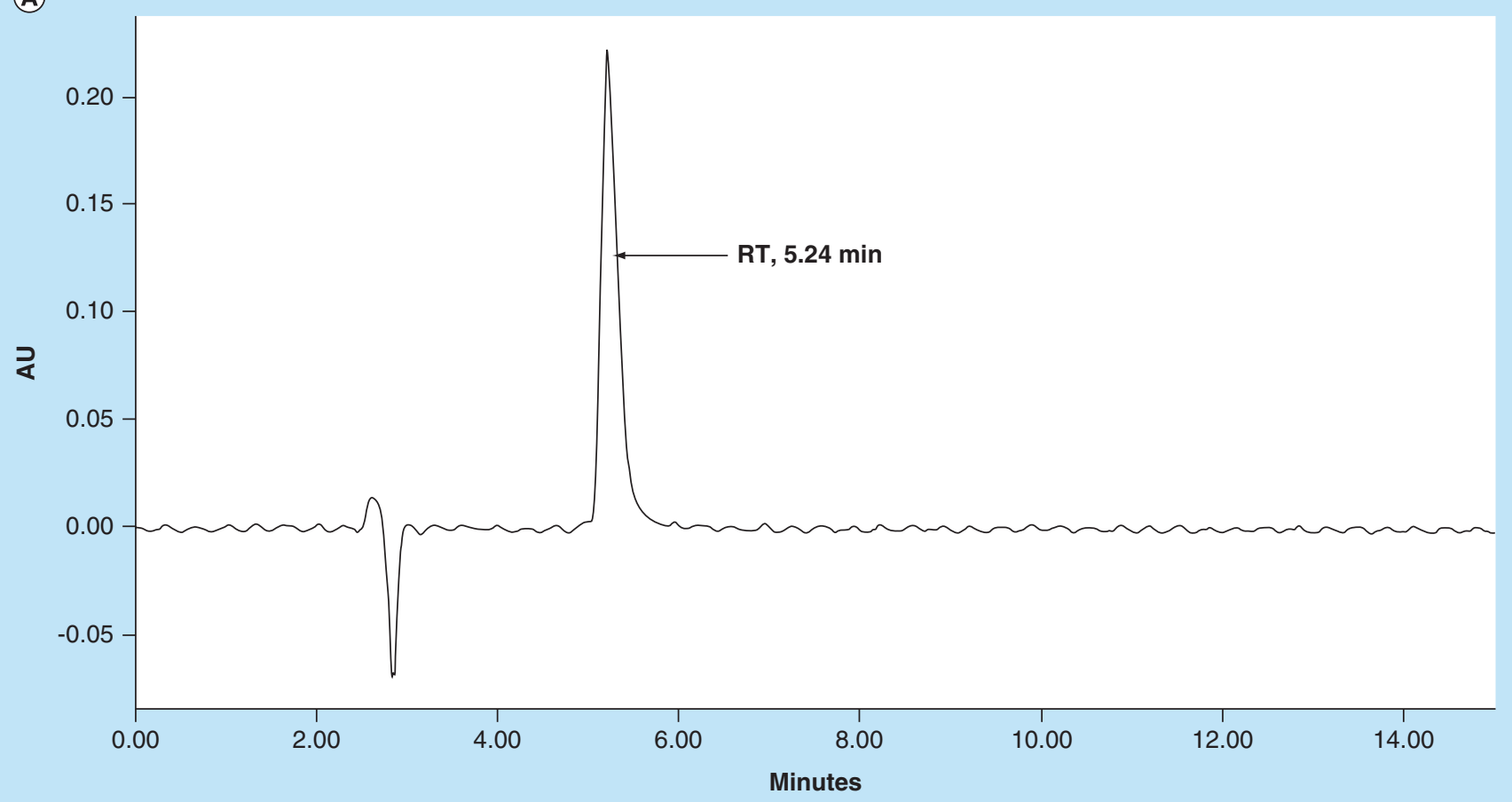

(B)

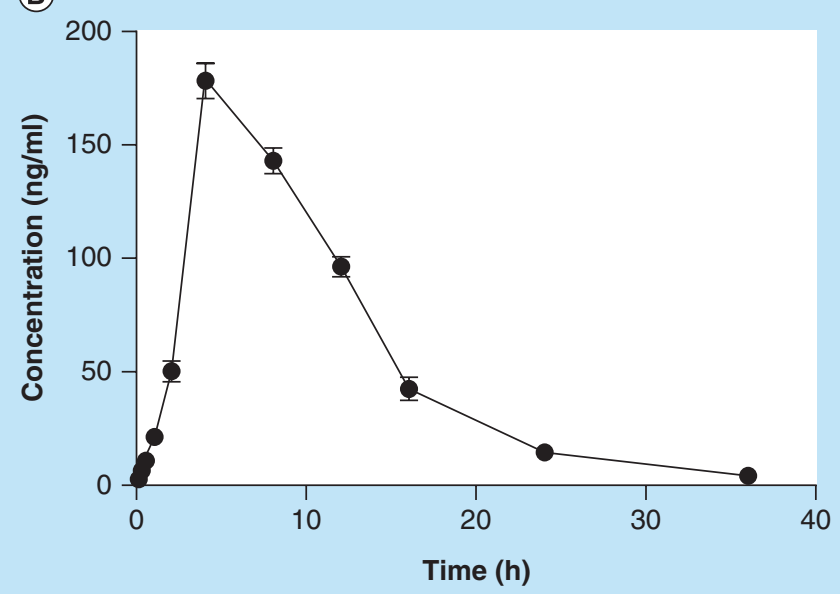

Figure 5. High-performance liquid chromatography chromatogram and drug concentration profile in rat plasma. (A) The retention time of M1. (B) Plasma drug concentration after single oral administration of M1 at $50 \mathrm{mg} / \mathrm{kg}$ dose at various time point $(\mathrm{n}=3$ ). RT: Retention time. 
M1 treatment in HCC rats. NAG protects the body from the oxidative stress because of anti-inflammatory and antioxidant properties. Thus, the increased levels NAG after M1 therapy might be related to suppress the inflammation and oxidative stress associated with hepatic injury in HCC rats.

Overall, the ameliorations of various metabolic markers after M1 treatment on HCC rats could be associated with the reconstruction of the cell membrane damages, improvement of the energy metabolism and repairing of the inflammation injury.

Further, the drug concentration in plasma was also measured to find out its absorption and tissue distribution. M1 had good $\mathrm{C}_{\max }(178.4 \mathrm{ng} / \mathrm{ml})$ which signified its good oral bioavailability and good plasma distribution. This result indicated that M1 had good absorption and, therefore, it showed its antiproliferative action on liver.

\section{Conclusion}

While searching for new HCC agents, our previous study unveiled that the MP seeds had good antiproliferative action on Huh-7 cells [8] and this plant is rich with isoquinoline alkaloid [9]. In another of our studies, one isoquinoline alkaloid, namely M1, isolated from MP seeds, showed good antiproliferative action in vitro on Huh-7 cells through 3-(4,5-dimethylthiazol-2-y1)2,5-diphenyl tetrazolium bromide (MTT) assay [12]. In the present study, in vivo antiproliferative effects of synthesized M1 in hepatocarcinogenic rats were investigated to evaluate whether the M1 therapy ameliorates the metabolic alterations caused by HCC and have protective action on liver tissue. The results suggested that M1 normalized various pathophysiological enzymes and increased antioxidant status, maintained normal tissue architecture and ameliorated metabolic profiling. The possible mechanisms could be related to reinstating the cell membrane damages, improving the energy metabolism and repairing the inflammation injury. HPLC analyses revealed that M1 had good plasma distribution after oral administration, signifying initiation of apoptosis in HCC. Altogether, this study provides the evidence that M1 might be effective against HCC.

\section{Future perspective}

Despite intense research, the immense potential of natural products in cancer therapy still remains unexplored. Consequently, there is a desideratum to develop more potent and less toxic anticancer drugs from plant-derived products. In view of this, a plant-derived natural product (M1) was synthesized and tested for in vivo anticancer efficacy on its two oral doses $-50 \mathrm{mg} / \mathrm{kg}$ and $100 \mathrm{mg} /$ $\mathrm{kg}$. Altogether, the study showed that M1 might emerge as one of the potential leads for future drug design. The next step for this research is to perform the in vivo anti- cancer efficacy at its higher safe dose - $250 \mathrm{mg} / \mathrm{kg}$ - and observe the changes in various molecular biomarkers inside the cells during HCC condition and after M1 treatment. Last, this lead compound (M1) needs to go through its toxicity profiling for the better clarification of its suitability for the treatment of liver cancer.

\section{Supplementary data}

To view the supplementary data that accompany this paper please visit the journal website at: www.future-science.com/ doi/full/10.4155/fsoa-2017-0008

\section{Author contributions}

S Saha designed study protocol. P Kumar, V Raj, A Rai and $S$ Saha performed pharmacological screening. AK Singh and S Saha performed synthesis of M1, docking and statistical data analysis. A Rawat, U Kumar, D Kumar and A Guleria performed NMR experiments and structure elucidation. A Guleria analyzed NMR-based metabolomics data and prepared the figures. A Guleria, S Saha and S Maity wrote the manuscript. All the authors reviewed the manuscript.

\section{Acknowledgements}

The authors acknowledge the Department of Medical Education, Govt. of Uttar Pradesh, for supporting the high-field NMR facility at the Centre of Biomedical Research, Lucknow, India.

\section{Financial \& competing interests disclosure}

S Saha expresses his thanks to the University Grants Commission (UGC), New Delhi, India, for providing UGC-MRP grant (Project no. 42-680/2013[SR]) and the Department of Science and Technology (DST) and Govt. of India (Ref. No. DST/SB/ EMEQ-320/2014). A Guleria acknowledges the DST and Govt. of India for financial assistance under DST INSPIRE Faculty Award (Ref. no. DST/Inspire Faculty Award 2014/LSBM-120). The authors have no other relevant affiliations or financial involvement with any organization or entity with a financial interest in or financial conflict with the subject matter or materials discussed in the manuscript apart from those disclosed.

No writing assistance was utilized in the production of this manuscript.

\section{Ethical conduct of research}

The authors state that they have obtained appropriate institutional review board approval or have followed the principles outlined in the Declaration of Helsinki for all human or animal experimental investigations. In addition, for investigations involving human subjects, informed consent has been obtained from the participants involved.

\section{Open access}

This work is licensed under the Creative Commons Attribution 4.0 License. To view a copy of this license, visit http://creativecommons.org/licenses/by/4.0/ 


\section{Summary points}

- Previously, an isoquinoline alkaloid (M1) isolated from Mucuna pruriens seeds was found effective against human hepatoma cell lines (Huh-7 cells) in vitro.

- In this experiment, M1 (6,7-dimethoxy-1,2,3,4-tetrahydro-isoquinoline-3-carboxylic acid) was synthesized and evaluated for in-vivo antiproliferative action in diethylnitrosamine-induced hepatocarcinogenic rats.

- Antioxidant study revealed the protective action of M1 on liver.

- Histopathology of liver displayed morphological changes and showed that M1 restored the arbitrary arrangement of liver tissues in normal proportion.

- Pharmacokinetic study through high-performance liquid chromatography analysis of plasma showed good oral bioavailability and plasma drug concentration after M1 administration.

- Nuclear magnetic resonance-based metabolic approach for hepatic carcinoma was employed to identify the alterations of metabolites/biomarkers during hepatic carcinoma and after M1 therapy. The study confirmed that M1 therapy ameliorated hepatocellular carcinoma-induced metabolic alterations which signified its antiproliferative potential.

\section{References}

1 Siegel R, Naishadham D, Jemal A. Cancer statistics, 2012. CA Cancer J. Clin. 62, 10-29 (2012).

2 Alwan A, MacLean DR, Riley LM et al. Monitoring and surveillance of chronic non-communicable diseases: progress and capacity in high-burden countries. Lancet 376, 1861-1868 (2011).

3 Gottesman MM, Pastan I. Biochemistry of multidrug resistance mediated by the multidrug transporter. Ann. Rev. Biochem. 62, 385-427 (1993).

4 Isnard-Bagnis C, Moulin B, Launay-Vacher V, Izzedine H, Tostivint I, Deray G. Renal toxicity of anticancer. Nephrol. Ther. 1, 101-114 (2005).

5 Smith BD, Bambach BJ, Vala MS et al. Inhibited apoptosis and drug resistance in acute myeloid leukaemia. Brit. J. Haematol. 102, 1042-1049 (1998).

6 Asghar U, Meyer T. Are there opportunities for chemotherapy in the treatment of hepatocellular cancer? J. Hepatol. 56, 686-695 (2012).

7 Ling CQ, Chiu JH, Oh B, Cho WCS. Natural products for liver diseases: basic, clinical, and translational research. Evid. Based Complementary Altern. Med. 2012, 794343 (2012).

8 Yadav RK, Singh M, Sarkar S, Maity S, Saha S. Cytotoxicity of different extracts of Mucuna pruriens seeds on hepatoma cells but not on normal hepatic cells. Pharmacogn. Commun. 5, 75-81 (2015).

9 Misra L, Wagner H. Alkaloidal constituents of Mucuna pruriens seeds. Phytochemistry 65, 2565-2567 (2004).

10 Maiti M, Kumar GS. Polymorphic nucleic acid binding of bioactive isoquinoline alkaloids and their role in cancer. J. Nucleic Acids. 2009, 593408 (2009).

11 Park HS, Kim EH, Kang MR, Chung IK, Cheong CJ, Lee WT. Spectroscopic studies on interaction of protoberberines with the deoxyoligonucleotide d (GCCGTCGTTTTACA) . Bull. Korean. Chem. Soc. 25, 1559-1563 (2004).

12 Kumar P, Rawat A, Keshari AK et al. Antiproliferative effect of isolated isoquinoline alkaloid from Mucuna pruriens seeds in hepatic carcinoma cells. Nat. Prod. Res. 30, 460-463 (2016).

13 Song S, Yuan P, Li P, Wu H, Lu J, Wei W. Dynamic analysis of tumor-associated immune cells in DEN-induced rat hepatocellular carcinoma. Int. Immunopharmacol. 22, 392-399 (2014).

14 Kushwaha PS, Raj V, Singh AK et al. Antidiabetic effects of isolated sterols from Ficus racemosa leaves. RSC Adv. 5, 35230-35237 (2015).

15 Keshari AK, Kumar G, Kushwaha PS et al. Isolated flavonoids from Ficus racemosa stem bark possess antidiabetic, hypolipidemic and protective effects in albino Wistar rats. J. Ethnopharmacol. 181, 252-262 (2016).

16 Lodhi RL, Maity S, Kumar P, Saraf SA, Kaithwas G, Saha S. Evaluation of mechanism of heaptotoxicity of leflunomide using abino Wistar rats. Afr. J. Pharm. Pharmacol. 7 , 1625-1631 (2013).

17 Saha S, Chan DSZ, Lee CY et al. Pyrrolidinediones reduce the toxicity of thiazolidinediones and modify their anti-diabetic and anti-cancer properties. Eur. J. Pharmacol. 697, 13-23 (2012).

18 Saha S, New LS, Ho HK, Chui WK, Chan ECY. Direct toxicity effects of sulfo-conjugated troglitazone on human hepatocytes. Toxicol. Lett. 195, 135-141 (2010).

19 Makos BK, Youson JH. Tissue levels of bilirubin and biliverdin in the sea lamprey, Petromyzon marinus $\mathrm{L}$, before and after biliary atresia. Comp. Biochem. Physiol. Part A Physiol. 91, 701-710 (1988).

20 Ong MM, Latchoumycandane C, Boelsterli UA. Troglitazoneinduced hepatic necrosis in an animal model of silent genetic mitochondrial abnormalities. Toxicol. Sci. 97, 205-213 (2007).

21 Wishart DS, Jewison T, Guo AC et al. HMDB 3.0 - the human metabolome database in 2013. Nucleic Acids Res. 41, D801-D807 (2013).

22 Nicholson JK, Foxall PJ, Spraul M, Farrant RD, Lindon JC. $750 \mathrm{MHz}{ }^{1} \mathrm{H}$ and ${ }^{1} \mathrm{H}-{ }^{13} \mathrm{C}$ NMR spectroscopy of human blood plasma. Anal. chem. 67, 793-811 (1995).

23 Guleria A, Bajpai NK, Rawat A, Khetrapal CL, Prasad $\mathrm{N}$, Kumar D. Metabolite characterisation in peritoneal dialysis effluent using highresolution ${ }^{1} \mathrm{H}$ and ${ }^{1} \mathrm{H}_{-}{ }^{13} \mathrm{C}$ NMR spectroscopy. Magnet. Reson. Chem. 52, 475-479 (2014).

24 MetaboAnalyst. www.metaboanalyst.ca/

25 Bhandari R, Kaur I. A sensitive HPLC method for determination of isoniazid in rat plasma, brain, liver and kidney. J. Chromatogr. Sep. Tech. 3, 1-5 (2012). 
26 Conte JE, Lin E, Zurlinden E. High-performance liquid chromatographic determination of pyrazinamide in human plasma, bronchoalveolar lavage fluid, and alveolar cells. J. Chromatogr. Sci. 38, 33-37 (2000).

27 McIntyre N, Rosalki S. Biochemical investigations in the management of liver disease. In: Hepatobiliary Diseases, Springer, NY, USA 39-71 (1992).

28 Saha S. Hepatotoxicity of Thiazolidinedione Antidiabetic Drugs: a Structural Toxicity Relationship Study [Ph. D. Thesis]. National University of Singapore, Singapore (2010).

29 Karaman A, Iraz M, Kirimlioglu H, Karadag N, Tas E, Fadillioglu E. Hepatic damage in biliary-obstructed rats is ameliorated by leflunomide treatment. Pediatr. Surg. Int. 22, 701-708 (2006).

30 Baudrimont I, Ahouandjivo R, Creppy E. Prevention of lipid peroxidation induced by ochratoxin A in Vero cells in culture by several agents. Chem. Biol. Interact. 104, 29-40 (1997).

31 Reiter RJ, Tan DX, Osuna C, Gitto E. Actions of melatonin in the reduction of oxidative stress. J. Biomed. Sci. 7 , 444-458 (2000).

32 Barrera G. Oxidative stress and lipid peroxidation products in cancer progression and therapy. ISRN Oncol. (2012), 137289 (2012)

33 Suzuki YJ, Carini M, Butterfield DA. Protein carbonylation. Antioxid. Redox. Signal. 12, 323-325 (2010).

34 Rigato I, Ostrow JD, Tiribelli C. Bilirubin and the risk of common non-hepatic diseases. Trends Mol. Med. 11, 277-283 (2005).

35 Ketterer B, Meyer D. Gluthathione transferases: a possible role in the detoxication and repair of DNA and lipid hydroperoxides. Mutat. Res. Fund. Mol. Mech. Mut. 214, 33-40 (1989).

36 Xia J, Sinelnikov IV, Han B, Wishart DS. MetaboAnalyst 3.0 - making metabolomics more meaningful. Nucleic Acids Res. 43(W1), 1-7 (2015).
37 Guleria A, Misra DP, Rawat A et al. NMR-based serum metabolomics discriminates takayasu arteritis from healthy individuals: a proof-of-principle study. J. Proteome. Res. 14, 3372-3381 (2015).

38 Liu Y, Hong Z, Tan G et al. NMR and LC/MS-based global metabolomics to identify serum biomarkers differentiating hepatocellular carcinoma from liver cirrhosis. Int. J. Cancer 135, 658-668 (2014).

39 Katz-Brull R, Seger D, Rivenson-Segal D, Rushkin E, Degani H. Metabolic markers of breast cancer: enhanced choline metabolism and reduced choline-etherphospholipid synthesis. Cancer Res. 62, 1966-1970 (2002).

40 Parrish WR, Rosas-Ballina M, Gallowitsch-Puerta M et al. Modulation of TNF release by choline requires $\alpha 7$ subunit nicotinic acetylcholine receptor-mediated signaling. Mol. Med. 14, 567-574 (2008).

41 Erol A. Retrograde regulation due to mitochondrial dysfunction may be an important mechanism for carcinogenesis. Med. Hypotheses 65, 525-529 (2005).

42 Gao H, Lu Q, Liu X et al. Application of ${ }^{1} \mathrm{H}$ NMR-based metabonomics in the study of metabolic profiling of human hepatocellular carcinoma and liver cirrhosis. Cancer Sci. 100, 782-785 (2009).

43 Huang Q, Tan Y, Yin P et al. Characterization of hepatocellular carcinoma using nontargeted tissue metabolomics. Cancer Res. 73, 4992-5002 (2013).

44 Fages A, Duarte-Salles T, Stepien M et al. Metabolomic profiles of hepatocellular carcinoma in a European prospective cohort. BMC Med. 13, 1-14 (2015).

45 Arnold JN, Saldova R, Hamid UM, Rudd PM. Evaluation of the serum $\mathrm{N}$-linked glycome for the diagnosis of cancer and chronic inflammation. Proteomics 8, 3284-3293 (2008). 